November 12, 1998

FERMILAB-PUB-98/357-T

\title{
Generic and Chiral Extensions of the Supersymmetric Standard Model
}

\author{
Hsin-Chia Cheng, Bogdan A. Dobrescu and Konstantin T. Matchev \\ Theoretical Physics Department \\ Fermi National Accelerator Laboratory \\ Batavia, Illinois, 60510, USA *
}

\begin{abstract}
We construct extensions of the Standard Model in which the gauge symmetries and supersymmetry prevent the dangerously large effects that may potentially be induced in a supersymmetric standard model by Planck scale physics. These include baryon number violation, flavor changing neutral currents, the $\mu$ term, and masses for singlet or vector-like fields under the Standard Model gauge group. For this purpose we introduce an extra non-anomalous $U(1)_{\mu}$ gauge group. Dynamical supersymmetry breaking in a secluded sector triggers the breaking of the $U(1)_{\mu}$ and generates soft masses for the superpartners via gauge mediation, with the scalars possibly receiving sizable contributions from the $U(1)_{\mu}$ D-term. We find several classes of complete and calculable models, in which the messengers do not present cosmological problems and neutrino masses can also be accomodated. We derive the sparticle spectrum in these models and study the phenomenological consequences. We give an exhaustive list of the potential experimental signatures and discuss their observability in the upcoming Tevatron runs. One class of models exhibits interesting new discovery channels, namely $W W E_{T}, W \gamma E_{T}^{\prime}$ and $W Z E_{T}^{\prime}$, which arise when the next-to-lightest supersymmetric particle is a short-lived $S U(2)_{W}$ neutralino.
\end{abstract}

*e-mail addresses: hcheng@fnal.gov, bdob@fnal.gov, matchev@fnal.gov 


\section{Introduction}

The gauge structure of the Standard Model (SM) has two important consequences in agreement with the experimental data: the proton stability is ensured by a discrete baryon number symmetry, and there are no tree-level flavor changing neutral currents (FCNC). On the other hand, a mass term for the Higgs doublet is not prevented by any symmetry in the Standard Model, leading to the well known hierarchy and naturalness problems.

The Minimal Supersymmetric Standard Model (MSSM) solves the naturalness problem (because supersymmetry [SUSY] ensures the cancellation of the quadratic divergences in the Higgs self-energy), but does not solve the hierarchy problem (i.e., it does not explain the large hierarchy between the $\mu$-term or the soft supersymmetry breaking parameters and the Planck scale). Moreover, the MSSM does not have the attractive features of the Standard Model mentioned above: the gauge structure allows both proton decay operators and FCNCs.

The resolution to these issues may be provided by physics beyond the MSSM. For example, the exponential hierarchy between the soft breaking parameters and the Planck scale is naturally produced if supersymmetry is dynamically broken. The tree level FCNCs are eliminated if there is a global $R$-symmetry, while radiative FCNCs can be kept sufficiently small if supersymmetry breaking is communicated to the MSSM fields by generation-independent gauge interactions. The proton decay operators can be avoided by invoking a discrete baryon number symmetry, and the $\mu$-term can be kept small compared with the Planck scale by a discrete symmetry whose breaking is triggered by the supersymmetry breaking. Likewise, some discrete symmetries may be used to eliminate other unacceptable operators associated with the new physics beyond the MSSM, such as large mass terms for the gauge singlets required by many gauge mediated supersymmetry breaking models.

At present, all viable supersymmetric extensions of the Standard Model rely on the existence of some discrete symmetries which are not known to be associated with gauge symmetries. This situation is rather unfortunate given that currently it is not known whether the global symmetries are preserved by quantum gravitational effects. In fact there are some arguments that support the idea that any global symmetry is badly violated in the presence of nonperturbative gravitational effects [1]: the global charges may leak through a wormhole, or they may disappear into a black hole which may evaporate. In the low energy effective theory, these effects give rise to gauge-invariant operators which 
break explicitly the global symmetries. Generically, one expects these operators to have coefficients of order one times the appropriate power of the Planck scale. This results in a $\mu$ term which is too large by 16 orders of magnitude, dimension-four baryon number violating operators which have coefficients 22 orders of magnitude larger than the upper bound set by the proton decay measurements, and other disastrous effects. However, in certain cases where general relativity is modified at energies significantly below the Planck scale [2], it is possible to suppress the coefficients of the symmetry violating operators. In any case, the extent of global symmetry violation appears to be highly sensitive to the underlying theory of quantum gravity, which is not known yet.

Hence, it would be useful to show that the global symmetries required in the MSSM are remnants of some spontaneously broken gauge symmetries. In string theory and M-theory there are situations where discrete symmetries in the low energy theory are remnants of gauge groups spontaneously broken by the string dynamics [3]. However, it is by no means clear that once the appropriate vacuum of a viable string theory is found, the necessary discrete symmetries of the MSSM would be preserved. Therefore, it has been often attempted to extend the SM gauge group so that the harmful operators allowed in the MSSM are no longer gauge invariant. The simplest extension is to include a spontaneously broken $U(1)$ gauge symmetry, and it has been used to avoid baryon number violating operators [4] or a large $\mu$-term [5]. Nevertheless, no chiral (i.e. without gauge invariant mass terms) and generic (i.e. without unnaturally small dimensionless couplings) supersymmetric model has been constructed yet.

In a previous paper [6] we showed that a new $U(1)$ gauge symmetry, in conjunction with supersymmetry and the standard $S U(3)_{C} \times S U(2)_{W} \times U(1)_{Y}$ gauge group, is sufficient to prevent any mass terms (including the $\mu$-term), so that the only fundamental dimensional parameter is the Planck scale. Although this is a chiral supersymmetric model, it relies as much as the MSSM on discrete symmetries to eliminate the proton decay operators. Given that our goal is to construct a self-consistent theory which does not invoke arbitrary assumptions about quantum gravity, we must use a gauge symmetry to eliminate the proton decay operators, as well as any other dimension-four and higher operators forbidden by phenomenology.

In this paper we show that the gauge group introduced in [6] is in fact sufficient to replace any discrete symmetry required by the phenomenological constraints, provided the charge assignments under the new $U(1)$ gauge symmetry are chosen carefully. We find several classes of phenomenologically viable models of this type. These are chiral 
and generic supersymmetric models to the extent that we do not attempt to explain the quark and lepton masses, so that we allow Yukawa couplings as small as $\sim \lambda_{e} \sim 10^{-5}$.

An interesting feature of our models is that the new $U(1)$ communicates supersymmetry breaking from a dynamical supersymmetry breaking (DSB) sector to the MSSM fields. Furthermore, unlike the previous models in which a spontaneously broken $U(1)$ mediates supersymmetry breaking [7, 8], the existence of a DSB sector and of a sector responsible for gaugino masses are required by the gauge anomaly cancellation conditions. As a consequence, the superpartner spectrum is quite distinctive. We discuss the resulting phenomenology and find some interesting cases with unexpected experimental signatures.

The plan of the paper is as follows. In Section 2 we discuss the theoretical and phenomenological constraints, and use them to find a fairly exhaustive class of viable models. In Section 3 we study the phenomenology of this class of models. We describe their low-energy spectrum and discuss the experimental search strategy in each of the typical scenarios, by singling out the most promising channels to look for in the upcoming Tevatron runs. The implications of relaxing some of the phenomenological constraints are considered in Section 4 , where we also draw our conclusions.

\section{Framework and Constraints}

If the gauge group acting on the MSSM chiral superfields is $S U(3)_{C} \times S U(2)_{W} \times U(1)_{Y} \times$ $U(1)_{\mu}$, then the $H_{u} H_{d}$ term in the superpotential is forbidden provided the $U(1)_{\mu}$ charges of the two Higgs superfields satisfy $z_{H_{u}}+z_{H_{d}} \neq 0$. In order to produce a Higgsino mass, we introduce a $S H_{u} H_{d}$ term in the superpotential, where the Standard Model singlet $S$ has $U(1)_{\mu}$ charge $z_{S}=-z_{H_{u}}-z_{H_{d}}$, and its scalar component acquires a vev.

In order to have quark and lepton masses and mixings (we allow lepton mixings in compliance with the recent Super-Kamiokande results [10]), the most general Yukawa couplings of the Higgs doublets to quarks and leptons require the $U(1)_{\mu}$ charges of the

quark and lepton superfields, $Q_{i}, \bar{U}_{i}, \bar{D}_{i}, L_{i}, \bar{E}_{i}, \nu_{R i}(i=1,2,3$ is a generational index), to be family-independent and to satisfy

$$
\begin{aligned}
& z_{Q}=-z_{H_{u}}-z_{\bar{U}}=-z_{H_{d}}-z_{\bar{D}} \\
& z_{L}=-z_{H_{u}}-z_{\nu}=-z_{H_{d}}-z_{\bar{E}} .
\end{aligned}
$$

These conditions can be relaxed if the quark and lepton mass matrices have textures produced by a non-standard mechanism, such as Frogatt-Nielsen [9], but we will not 
study this possibility here.

For $U(1)_{\mu}$ to be anomaly free, additional chiral superfields have to be included. The $\left[S U(3)_{C}\right]^{2} \times U(1)_{\mu},\left[S U(2)_{W}\right]^{2} \times U(1)_{\mu},\left[U(1)_{Y}\right]^{2} \times U(1)_{\mu}$ anomalies from the MSSM fields are円

$$
\begin{aligned}
(A 3) & \equiv\left[S U(3)_{C}\right]^{2} \times U(1)_{\mu}: \quad 3\left(2 z_{Q}+z_{\bar{U}}+z_{\bar{D}}\right)=3 z_{S}, \\
(A 2) & \equiv\left[S U(2)_{W}\right]^{2} \times U(1)_{\mu}: \quad 9 z_{Q}+3 z_{L}-z_{S}, \\
(A 1) & \equiv\left[U(1)_{Y}\right]^{2} \times U(1)_{\mu}: \quad-9 z_{Q}-3 z_{L}+7 z_{S} .
\end{aligned}
$$

They have to be cancelled by fields which carry both $\mathrm{SM}$ and $U(1)_{\mu}$ quantum numbers. In order not to introduce anomalies to the SM gauge group, and to be able to decouple at low energies after $U(1)_{\mu}$ is broken, these fields should be vector-like under the SM gauge group. As a result, they can naturally be identified with the messengers of gauge mediated supersymmetry breaking.

The masses of the messengers are induced by a $X \phi \bar{\phi}$ term in the superpotential, where $\phi, \bar{\phi}$ represent the messenger fields, $X$ is a SM singlet, and their $U(1)_{\mu}$ charges are related by $z_{\phi}+z_{\bar{\phi}}=-z_{X}$.

In order to generate the soft supersymmetry breaking masses for the MSSM fields through gauge mediation, the $X$ superfield should have both scalar and $F$-type vevs with $\left\langle F_{X}\right\rangle /\langle X\rangle \sim 10^{4}-10^{5} \mathrm{GeV}$ and hence can not be identified with the $S$ field (otherwise it will give a too big $B$ term for the Higgs sector). The simplest way to have a (local) minimum in which $S$ and $X$ obtain the desired vevs is having only one $X$ field which couples to all messengers, and introducing another SM singlet $N$, with the superpotential in Ref. 6],

$$
W=f X \phi \bar{\phi}+\frac{\lambda}{2} X N^{2}-\frac{\epsilon}{2} S N^{2}+\kappa S H_{u} H_{d}
$$

Phenomenological contraints require $\lambda^{3 / 2}<\epsilon \ll \lambda \ll f \sim 1$ [6]. For $\kappa>\sqrt{\lambda^{2}+\epsilon^{2}}$, there is a desired minimum in which all $S, X$, and $N$ fields obtain vevs, after they receive negative masses squared of their scalar components from the DSB sector [6]. This choice of superpotential imposes the following relation between the $U(1)_{\mu}$ charges of $S, X$, and $N$ fields

$$
z_{S}=z_{X}=-2 z_{N}
$$

There are two other possible terms in the superpotential, which are allowed by the gauge symmetries, $f^{\prime} S \phi \bar{\phi}$ and $\kappa^{\prime} X H_{u} H_{d}$. The minimum will not be affected if the $\kappa^{\prime}$ coupling is

\footnotetext{
${ }^{1}$ We use the normalization $\operatorname{tr}\left(T^{c}\left\{T^{a}, T^{b}\right\}\right)$ for the anomalies, so the $\left[U(1)_{Y}\right]^{2} \times U(1)_{\mu}$ anomaly from a field with $U(1)_{Y} \times U(1)_{\mu}$ charges $(y, z)$ is $2 y^{2} z$.
} 
small. The first term contributes to the messenger masses and the second term gives extra contribution to the $B$ term of the Higgs sector. We assume that the couplings $f^{\prime}$ and $\kappa^{\prime}$, if they exist, are small so that the messenger masses receive dominant contributions from $X$ and the desired minimum is not destablized.

For a vector-like pair of messengers $\phi, \bar{\phi}$ with $S U(3)_{C}$ index $T_{3 \phi}=\left(T_{3 \bar{\phi}}\right)$, (normalized to $1 / 2$ for the fundamental representation), $S U(2)_{W}$ index $T_{2 \phi}$, and $U(1)_{Y}$ charges $\pm y_{\phi}$, the contributions to the anomalies (A3)-(A1) are

$$
\begin{aligned}
& 2 T_{3 \phi}\left(z_{\phi}+z_{\bar{\phi}}\right)=-2 T_{3 \phi} z_{X}, \\
& 2 T_{2 \phi}\left(z_{\phi}+z_{\bar{\phi}}\right)=-2 T_{2 \phi} z_{X}, \\
& 2 y_{\phi}^{2}\left(z_{\phi}+z_{\bar{\phi}}\right)=-2 y_{\phi}^{2} z_{X} .
\end{aligned}
$$

A messenger field, $a$, which is real under SM with $U(1)_{\mu}$ charge $-z_{X} / 2$ can obtain its mass from the vev of $X$ without its conjugate partner. In this case, its contributions to (A3)-(A1) are $-T_{3 a} z_{X},-T_{2 a} z_{X}$, and $-y_{a}^{2} z_{X}$, respectively.

To cancel the anomalies coming from the MSSM sector [eq. (2.2)], the messengers have to satisfy

$$
\begin{array}{r}
3 z_{S}-\sum_{r_{3}} T_{3 r_{3}} z_{S}=0 \\
9 z_{Q}+3 z_{L}-z_{S}-\sum_{r_{2}} T_{2 r_{2}} z_{S}=0 \\
-9 z_{Q}-3 z_{L}+7 z_{S}-\sum_{r_{1}} y_{r_{1}}^{2} z_{S}=0
\end{array}
$$

where $r_{i}$ runs over all messenger representations (counting the SM vector-like pair separately) under $S U(3)_{C}, S U(2)_{W}$, and $U(1)_{Y}$ respectively. The gauge mediated contributions to the soft masses of the MSSM fields transforming under $S U(3)_{C}, S U(2)_{W}$, and $U(1)_{Y}$ are proportional to the messenger multiplicity factors

$$
\Delta \beta_{3} \equiv \sum_{r_{3}} T_{3 r_{3}}, \quad \Delta \beta_{2} \equiv \sum_{r_{2}} T_{2 r_{2}}, \quad \Delta \beta_{1} \equiv \sum_{r_{1}} y_{r_{1}}^{2}
$$

which are just the changes of the one-loop $\beta$-function coefficients of the corresponding gauge groups due to the messenger fields. From eq. (2.6) we see that

$$
\Delta \beta_{3}=\sum_{r_{3}} T_{3 r_{3}}=3
$$

which means the messenger sector should either contain three pairs of $\mathbf{3}$ and $\overline{\mathbf{3}}$, or one 8 under $S U(3)_{C}$. Combining eqs. (2.7) and (2.8) we obtain another constraint on the 
messenger sector,

$$
\Delta \beta_{2}+\Delta \beta_{1}=\sum_{r_{2}} T_{2 r_{2}}+\sum_{r_{1}} y_{r_{1}}^{2}=6,
$$

which limits $\Delta \beta_{2}$ and $\Delta \beta_{1}$ to a discrete set of choices.

The only possible messengers which can satisfy eqs. (2.10) and (2.11) (and do not cause the SM gauge couplings blowing up below the Planck scale) are the ones transforming under $S U(3)_{C} \times S U(2)_{W}$ as $(\mathbf{3}, \mathbf{2}),(\mathbf{3}, \mathbf{1}),(\mathbf{8}, \mathbf{1}),(\mathbf{1}, \mathbf{2}),(\mathbf{1}, \mathbf{3}),(\mathbf{1}, \mathbf{1})$ and their conjugates. If they have arbitrary hypercharges, then in general they can not decay into MSSM fields. They will be stable and form bound states with fractional electric charges, which may cause cosmological problems unless a late period of inflation is incorporated. To avoid that, the hypercharges of the messenger fields are fixed up to additive integers by the hypercharges of the MSSM fields. Imposing the conditions (2.10) and (2.11), we find that the messenger sector can only consist of fields among $q=(\mathbf{3}, \mathbf{2},+1 / 6), \bar{u}=$ $(\overline{\mathbf{3}}, \mathbf{1},-2 / 3), \bar{d}=(\overline{\mathbf{3}}, \mathbf{1},+1 / 3), a=(\mathbf{8}, \mathbf{1}, 0), l=(\mathbf{1}, \mathbf{2},-1 / 2), w=(\mathbf{1}, \mathbf{3}, 0), \bar{e}=(\mathbf{1}, \mathbf{1},+1)$, and their conjugates. There are 16 possible combinations with four different sets of $\left(\Delta \beta_{3}, \Delta \beta_{2}, \Delta \beta_{1}\right)$, which are shown in Table 1 .

Already from the above simple constraints, we can see that there are only four possible combinations of the gauge mediated contributions to the soft masses of the MSSM fields. In particular for the SM gaugino masses, which only receive masses from gauge mediation, their ratios are fixed to these four cases, independent of the assumption that there are no states with fractional electric charges. If the $U(1)_{\mu} D$ term and the other contributions are small compared to the gauge mediated contributions, then the complete sparticle spectrum is determined to a large extent in these four cases. For larger $U(1)_{\mu} D$ term contributions, we also need to know the specific $U(1)_{\mu}$ charges of the MSSM fields, in order to predict the scalar superpartner spectrum.

In addition to (2.6) $-(2.8)$, the $U(1)_{\mu}$ charges also have to satisfy the $U(1)_{Y} \times\left[U(1)_{\mu}\right]^{2}$, $U(1)_{\mu}$, and $\left[U(1)_{\mu}\right]^{3}$ anomaly cancellation conditions. In general, the latter two anomalies are not cancelled by the combination of the MSSM and messenger sector. Therefore, some fields from the DSB sector have to carry $U(1)_{\mu}$ charges, so that $U(1)_{\mu}$ can communicate supersymmetry breaking to both the messenger sector and to the MSSM chiral superfields. It is remarkable that the existence of the three sectors (MSSM, messenger and DSB) is required by the mathematical consistency of the theory (namely the anomaly cancellation conditions). We consider this situation an improvement compared with the original gaugemediated models [12, 11] in which the three different sectors are introduced only for phenomenological reasons. 


\begin{tabular}{|c||c|c|c|c|c|c|c||c|c|c|}
\hline Model & $d \bar{d}$ & $u \bar{u}$ & $q \bar{q}$ & $a$ & $\bar{l}$ & $w$ & $e \bar{e}$ & $\Delta \beta_{3}$ & $\Delta \beta_{2}$ & $\frac{3}{5} \Delta \beta_{1}$ \\
\hline \hline $1 \mathrm{a}$ & 3 & - & - & - & 1 & - & 1 & 3 & 1 & 3 \\
\hline $1 \mathrm{~b}$ & 2 & 1 & - & - & 1 & - & - & 3 & 1 & 3 \\
\hline $1 \mathrm{c}$ & - & - & - & 1 & 1 & - & 2 & 3 & 1 & 3 \\
\hline \hline $2 \mathrm{a}$ & 3 & - & - & - & 2 & - & - & 3 & 2 & 2.4 \\
\hline $2 \mathrm{~b}$ & 3 & - & - & - & - & 1 & 1 & 3 & 2 & 2.4 \\
\hline $2 \mathrm{c}$ & 2 & 1 & - & - & - & 1 & - & 3 & 2 & 2.4 \\
\hline $2 \mathrm{~d}$ & - & - & - & 1 & 2 & - & 1 & 3 & 2 & 2.4 \\
\hline $2 \mathrm{e}$ & - & - & - & 1 & - & 1 & 2 & 3 & 2 & 2.4 \\
\hline \hline $3 \mathrm{a}$ & 3 & - & - & - & 1 & 1 & - & 3 & 3 & 1.8 \\
\hline $3 \mathrm{~b}$ & 1 & - & 1 & - & - & - & 1 & 3 & 3 & 1.8 \\
\hline $3 \mathrm{c}$ & - & 1 & 1 & - & - & - & - & 3 & 3 & 1.8 \\
\hline $3 \mathrm{~d}$ & - & - & - & 1 & 3 & - & - & 3 & 3 & 1.8 \\
\hline \hline 4a & 3 & - & - & - & - & 2 & - & 3 & 4 & 1.2 \\
\hline $4 \mathrm{~b}$ & 1 & - & 1 & - & 1 & - & - & 3 & 4 & 1.2 \\
\hline $4 \mathrm{c}$ & - & - & - & 1 & 2 & 1 & - & 3 & 4 & 1.2 \\
\hline $4 \mathrm{~d}$ & - & - & - & 1 & - & 2 & 1 & 3 & 4 & 1.2 \\
\hline
\end{tabular}

Table 1: Possible number of messenger representations, and the corresponding contributions to the gauge coupling beta functions. The factor of $3 / 5$ in front of $\Delta \beta_{1}$ corresponds to the $S U(5)$ normalization of the hypercharge.

If the DSB sector dynamics does not break $U(1)_{\mu}$, then its contributions to the $U(1)_{\mu}$ and $\left[U(1)_{\mu}\right]^{3}$ anomalies can be represented by low energy effective composite degrees of freedom a la 't Hooft [13]. The simplest example is the 3-2 model [14, 12], where after $S U(3)$ becomes strong and breaks supersymmetry, there is one light field charged under the unbroken "messenger" $U(1)$. Other DSB models have a different number of light composite fields with various $U(1)_{\mu}$ charge ratios. For simplicity, in searching for solutions, we restrict ourselves to the cases with no more than 2 extra such SM neutral and $U(1)_{\mu}$ charged composite fields from the DSB sector. A renormalizable and calculable example of a DSB model which gives rise to two light $U(1)_{\mu}$ charged composite fields is 
the $S U(4) \times S U(3)$ model [15, 16, 6]. A brief description of the model and its $U(1)_{\mu}$ charge assignments is presented in Appendix A.

There are several additional constraints we impose when we search for models. We allow the right-handed neutrinos to acquire Majorana masses, so the $U(1)_{\mu}$ charges of the right-handed neutrinos have to be $z_{\nu}=-z_{S} / 2$ or $-z_{N} / 2$ if they receive masses from $S$ or $N$ vevs. Note that we avoid $z_{\nu}=0$ because in that case the field content would not be chiral: the right-handed neutrinos would be gauge singlets, and a Planck scale mass for $L_{i}$ and $H_{u}$ would be potentially induced. For $z_{\nu}=-z_{S} / 2\left(=z_{N}\right)$, the operators $N L_{i} H_{u}$ are gauge invariant, and give rise to the bilinear $R$ parity violating terms after $N$ acquires a vev. The phenomenological constraints on these bilinear terms (e.g., from flavor changing neutral currents) require the couplings of the $N L_{i} H_{u}$ interactions to be very small. We will therefore only concentrate on the case $z_{\nu}=-z_{N} / 2$. In this case we will find that $R$ parity conservation is an automatic consequence of the gauge symmetry.

We are free to choose $z_{S}>0$ (note that $z_{S} \neq 0$ to avoid a large $\mu$ term), which implies that the $U(1)_{\mu} D$ term is positive. We will require the $U(1)_{\mu}$ charges for ordinary quarks and leptons to be non-negative, so that they do not receive negative masses squared from the $U(1)_{\mu} D$ term. This may not be necessary if the positive contributions from gauge mediation are larger than the negative $D$ term contributions. However, the squarks and sleptons receive $D$ term masses at a higher scale, so the SM gauge group may be broken before the gauge mediated contributions can turn on. Therefore, we do not search for models with negative quark or lepton charges.

Finally, if the messenger fields do not couple to the MSSM fields, they are stable. For typical values of the messenger masses, they will overclose the universe [17], unless diluted by a late period of inflation. We therefore require that the $U(1)_{\mu}$ charges allow the messenger fields to couple to the MSSM fields so that the messenger fields can decay into MSSM fields before nucleosynthesis. This requires the relevant matter-messenger couplings to be suppressed by no more than one power of the Planck mass [17. At the same time, the matter-messenger interactions which can induce too fast proton decays should be forbidden (including the lepton number conserving decays to gravitinos [18]).

The $U(1)_{\mu}$ charges of the MSSM fields can be expressed in terms of the 4 charges, $z_{Q}, z_{L}, z_{H_{u}}$, and $z_{S}$, from the requirements of the MSSM superpotential interactions. The Majorana masses of the right-handed neutrinos impose a relation among $z_{L}, z_{H_{u}}$, and $z_{S}\left(-z_{L}-z_{H_{u}}=z_{\nu}=z_{S} / 4\right)$. Among the anomaly conditions (2.6)-(2.8), only 2 combinations have been used. The other one, which can be taken as (2.7), gives another 
constraint among $z_{Q}, z_{L}$, and $z_{S}$ for each choice of $\Delta \beta_{2}$,

$$
9 z_{Q}+3 z_{L}-\left(1+\Delta \beta_{2}\right) z_{S}=0
$$

We choose the overall charge normalization by fixing $z_{S}$. The $U(1)_{\mu}$ charges of the MSSM fields then depend only on one independent charge, for example $z_{Q}$, and its range is limited by the requirement that the quark and lepton $U(1)_{\mu}$ charges are non-negative. The $U(1)_{\mu}$ charges of the MSSM fields as a function of $z_{Q}$, and the allowed range for $z_{Q}$ for each case of $\Delta \beta_{2}$ are shown in Tables 2 and 3 , respectively.

\begin{tabular}{|c|c|}
\hline$Q_{i}$ & $z_{Q}$ \\
\hline $\bar{U}_{i}$ & $-4 z_{Q}+5+4\left(\Delta \beta_{2}-2\right) / 3$ \\
\hline $\bar{D}_{i}$ & $2 z_{Q}-1-4\left(\Delta \beta_{2}-2\right) / 3$ \\
\hline$L_{i}$ & $-3 z_{Q}+4+4\left(\Delta \beta_{2}-2\right) / 3$ \\
\hline $\bar{E}_{i}$ & $6 z_{Q}-5-8\left(\Delta \beta_{2}-2\right) / 3$ \\
\hline$\nu_{i}$ & 1 \\
\hline$H_{u}$ & $3 z_{Q}-5-4\left(\Delta \beta_{2}-2\right) / 3$ \\
\hline$H_{d}$ & $-3 z_{Q}+1+4\left(\Delta \beta_{2}-2\right) / 3$ \\
\hline
\end{tabular}

Table 2: $U(1)_{\mu}$ charges of the MSSM fields in terms of $z_{Q}$, with the normalization $z_{S}=4$.

\begin{tabular}{|l|l|}
\hline$\Delta \beta_{2}=1$ & $14 / 36 \leq z_{Q} \leq 32 / 36$ \\
\hline$\Delta \beta_{2}=2$ & $30 / 36 \leq z_{Q} \leq 45 / 36$ \\
\hline$\Delta \beta_{2}=3$ & $46 / 36 \leq z_{Q} \leq 57 / 36$ \\
\hline$\Delta \beta_{2}=4$ & $66 / 36 \leq z_{Q} \leq 69 / 36$ \\
\hline
\end{tabular}

Table 3: The range of $z_{Q}$ for all MSSM quark and lepton charges being non-negative, normalizing to $z_{S}=4$.

For the cases in Table 1 and "reasonably simple" $U(1)_{\mu}$ charges in the corresponding allowed range, we search numerically for the messenger and (DSB sector composite) singlet charges which satisfy the rest of the anomaly constraints, allow messengers to decay 
fast enough, and forbid too rapid proton decay. Some of the solutions satisfying all the constraints are listed in Table $₫$. The fields $b_{1,2}$ are the light composite superfields from the DSB sector which carry $U(1)_{\mu}$ charges. Note that mass terms involving $b_{1,2}$ and $S$ or $X$ can be generated only by higher dimensional operators involving the fundamental fields from the DSB, and therefore are Planck-scale suppressed. We find solutions in only a few out of the 16 cases because of the restriction that there are no more than two $b_{i}$ superfields. If we relax this simplifying assumption and allow more singlets, there could be solutions in other cases as well.

The low energy MSSM spectrum and phenomenology depend mainly on $\Delta \beta_{1,2,3}$ and the $U(1)_{\mu}$ charges of the MSSM fields. They have little dependence on the exact compositions and charge assignments of the mesenger and DSB sectors as long as the mixings between the MSSM fields and messenger fields are small. We will discuss the phenomenology in the next section.

\section{Phenomenology}

\subsection{Particle spectrum}

First we shall briefly review the parameter space of this class of models and discuss the possible particle spectra arising in each case. For the rest of this section, we shall use the $U(1)_{\mu}$ charge normalization $z_{S}=4$ and rescale the charges in Table 4 correspondingly.

The desired minimum of the potential is at $\left\langle H_{u}\right\rangle=\left\langle H_{d}\right\rangle=0$ (at the scale of $U(1)_{\mu}$ breaking) and

$$
\left\langle N^{2}\right\rangle=\frac{24 \tilde{m}^{2}}{\lambda^{2}+\epsilon^{2}}, \quad\langle X\rangle=\frac{\epsilon}{\lambda}\langle S\rangle, \quad\left\langle S^{2}\right\rangle=\frac{\lambda^{2}}{\lambda^{2}+\epsilon^{2}}\left(\frac{\xi^{2}}{4}+\frac{\tilde{m}^{2}}{g_{\mu}^{2}}+\frac{12 \tilde{m}^{2}}{\lambda^{2}+\epsilon^{2}}\right) .
$$

The corresponding SUSY-breaking $F$ and $D$-terms are induced at the $U(1)_{\mu}$ breaking scale

$$
M_{\mu} \equiv g_{\mu}\langle N\rangle \simeq 2 \sqrt{6} \frac{g_{\mu}}{\lambda} \tilde{m} \quad(\gg \tilde{m})
$$

where $g_{\mu}$ is the $U(1)_{\mu}$ gauge coupling, and are given by

$$
\left\langle F_{N}\right\rangle=0, \quad\left\langle F_{X}\right\rangle=\frac{\lambda}{2}\left\langle N^{2}\right\rangle \simeq \sqrt{6} \tilde{m}\langle N\rangle, \quad\left\langle F_{S}\right\rangle=-\frac{\epsilon}{2}\left\langle N^{2}\right\rangle, \quad g_{\mu}^{2}\langle D\rangle=4 \tilde{m}^{2}
$$

The $\langle X\rangle$ and $\left\langle F_{X}\right\rangle$ vevs provide the SUSY preserving and breaking masses for the messenger fields $\phi$ and $\bar{\phi}$. The gauge singlets $X, S$ and $N$ also get masses. Their fermionic

\footnotetext{
${ }^{2}$ For more details, we refer the reader to Ref. [6].
} 


\begin{tabular}{|c|c|c|c|c|c|}
\hline \multirow[t]{2}{*}{ Fields } & \multicolumn{5}{|c|}{ Models } \\
\hline & 1a(i) & $2 \mathrm{a}(\mathrm{i})$ & 2a(ii) & $2 \mathrm{a}(\mathrm{iii})$ & $2 \mathrm{a}(\mathrm{iv})$ \\
\hline$Q$ & 2 & 8 & 1 & 10 & 11 \\
\hline $\bar{U}$ & 3 & 13 & 1 & 5 & 1 \\
\hline $\bar{D}$ & 5 & 7 & 1 & 11 & 13 \\
\hline$L$ & 2 & 12 & 1 & 6 & 3 \\
\hline $\bar{E}$ & 5 & 3 & 1 & 15 & 21 \\
\hline$\nu_{R}$ & 3 & 9 & 1 & 9 & 9 \\
\hline$H_{u}$ & -5 & -21 & -2 & -15 & -12 \\
\hline$H_{d}$ & -7 & -15 & -2 & -21 & -24 \\
\hline$S, X$ & 12 & 36 & 4 & 36 & 36 \\
\hline$N$ & -6 & -18 & -2 & -18 & -18 \\
\hline $\bar{d}_{i}$ & -4 & -2 & 0 & 2 & 4 \\
\hline$d_{i}$ & -8 & -34 & -4 & -38 & -40 \\
\hline$l_{i}$ & -1 & 12 & 1 & 6 & 3 \\
\hline $\bar{l}_{i}$ & -11 & -48 & -5 & -42 & -39 \\
\hline $\bar{e}$ & -4 & - & - & - & - \\
\hline$e$ & -8 & - & - & - & - \\
\hline$b_{1}$ & -12 & -39 & -4 & -36 & -36 \\
\hline$b_{2}$ & 18 & 90 & 10 & 90 & 90 \\
\hline $\begin{array}{c}\text { Messenger } \\
\text { decay } \\
\text { operators }\end{array}$ & $\begin{array}{c}Q L \bar{d}, \bar{U} \bar{E} d, \\
\bar{D} \nu_{R} d, L L \bar{e} \\
\bar{E} \nu_{R} e, N Q \bar{D} l \\
N L \bar{E} l, \bar{E} \nu_{R} H_{d} l, \\
X N H_{u} l, \nu_{R} \nu_{R} H_{u} l\end{array}$ & & $\begin{array}{r}\bar{E} H_{d} l, \\
X \\
N \\
N i \\
Q \nu\end{array}$ & $\begin{array}{l}\nu_{R} H_{u}, l \\
L \bar{l} \\
L \bar{d} \\
\bar{D} \bar{d} \\
H_{d} \bar{d}\end{array}$ & \\
\hline
\end{tabular}

Table 4: Solutions for the $U(1)_{\mu}$ charges (normalized to integers), which satisfy all the constraints. In models $1 \mathrm{a}(\mathrm{i})$ and $2 \mathrm{a}(\mathrm{ii})$ we find many other possible solutions with different messenger charges, including different charges for the different $d_{i}$ 's and $l_{i}$ 's. Here we only list one example for each case. 
components mix with the $U(1)_{\mu}$ gaugino to form two Dirac fermions, with masses $\sim$ $24\left(g_{\mu} / \lambda\right) \tilde{m}$ and $\sim 4 \tilde{m}$, respectively. The scalar components of the singlets also mix, and the resulting mass spectrum consists of a massless Nambu-Goldstone boson, eaten by the $U(1)_{\mu}$ gauge boson; a scalar of mass $24\left(g_{\mu} / \lambda\right) \tilde{m}$, which becomes part of the heavy gauge supermultiplet; and four light scalars with masses $2 \sqrt{6} \tilde{m}, 2 \sqrt{6} \tilde{m}, 2 \sqrt{3} \tilde{m}$ and $2 \sqrt{2} \tilde{m}$, correspondingly [6].

Assuming $\kappa^{\prime}=0$ for the moment, $\langle S\rangle$ and $\left\langle F_{S}\right\rangle$ provide the $\mu$ and $B$ terms for the Higgs sector:

$$
\begin{gathered}
\mu\left(M_{\mu}\right)=\kappa\langle S\rangle \simeq 2 \sqrt{3} \frac{\kappa}{\lambda} \tilde{m} \quad(\gtrsim \tilde{m}), \\
B\left(M_{\mu}\right)=\frac{\left\langle F_{S}\right\rangle}{\langle S\rangle} \simeq-2 \sqrt{3} \frac{\epsilon}{\lambda} \tilde{m} \quad(|B| \ll \tilde{m}) .
\end{gathered}
$$

Below the messenger scale

$$
M \equiv f\langle X\rangle \simeq 2 \sqrt{3} \frac{\epsilon f}{\lambda^{2}} \tilde{m} \quad(\gg \tilde{m})
$$

the messengers are integrated out, giving rise to the usual one-loop gauge mediation contributions to the gaugino masses:

$$
M_{n}(M)=\Delta \beta_{n} \frac{\alpha_{n}}{4 \pi} \Lambda g(\Lambda / M),
$$

where $n=1,2,3$ corresponds to $U(1)_{Y}, S U(2)_{W}$ and $S U(3)_{C}, g(x)$ is the threshold function from [19] and

$$
\Lambda \equiv \frac{\left\langle F_{X}\right\rangle}{\langle X\rangle} \simeq 2 \sqrt{3} \frac{\lambda}{\epsilon} \tilde{m}
$$

The scalar squared masses receive a $U(1)_{\mu} D$-term contribution and a negative contribution from the $U(1)_{\mu}$ mediation:

$$
m_{\tilde{f}}^{2}\left(M_{\mu}\right)=z_{f}\left(4-z_{f}\right) \tilde{m}^{2},
$$

in addition to the usual two-loop SM gauge mediation contributions:

$$
m_{\tilde{f}}^{2}(M)=\frac{2 \Lambda^{2}}{(4 \pi)^{2}}\left(\Delta \beta_{3} C_{3}^{f} \alpha_{3}^{2}+\Delta \beta_{2} C_{2}^{f} \alpha_{2}^{2}+\frac{5}{3} \Delta \beta_{1} C_{1}^{f} \alpha_{1}^{2}\right) f(\Lambda / M),
$$

where the coefficients $C_{i}^{f}$ are zero for gauge singlet sfermions $\tilde{f}$, and $4 / 3,3 / 4$ and $y^{2}$ for fundamental representations of $S U(3)_{C}, S U(2)_{W}$ and $U(1)_{Y}$, correspondingly. The threshold function $f(x)$ can be found in Ref. [19]. 
After imposing electroweak symmetry breaking, the parameter space of this class of models is spanned by $\left\{\Lambda, M, M_{\mu}, \tan \beta, \operatorname{sign}(\mu)\right\}$. However, if we allow a small coupling $\kappa^{\prime} X H_{u} H_{d}$, the conditions (3.4) and (3.5) can be relaxed:

$$
\begin{gathered}
\mu\left(M_{\mu}\right)=\kappa\langle S\rangle+\kappa^{\prime}\langle X\rangle \simeq 2 \sqrt{3}\left(\frac{\kappa}{\lambda}+\frac{\kappa^{\prime} \epsilon}{\lambda^{2}}\right) \tilde{m} \quad(\gtrsim \tilde{m}), \\
B\left(M_{\mu}\right)=\frac{\kappa\left\langle F_{S}\right\rangle+\kappa^{\prime}\left\langle F_{X}\right\rangle}{\kappa\langle S\rangle+\kappa^{\prime}\langle X\rangle} \simeq-2 \sqrt{3}\left(\frac{\epsilon}{\lambda}+\frac{\kappa^{\prime}}{\kappa}\right) \tilde{m} \quad(|B| \lesssim \tilde{m}),
\end{gathered}
$$

so that $\tilde{m}$ can be traded for $\kappa^{\prime} / \lambda$ and treated as an additional free parameter. This is particularly relevant for models with $z_{H_{d}}<z_{H_{u}}$, where it is rather difficult to obtain proper electroweak symmetry breaking at large values of $\tan \beta$, which are suggested by (3.5). This can be easily understood as follows. Minimization of the tree-level potential leads to the approximate relation

$$
m_{H_{d}}^{2}\left(M_{Z}\right)-m_{H_{u}}^{2}\left(M_{Z}\right) \simeq m_{A}^{2}\left(M_{Z}\right)
$$

which implies that $m_{H_{d}}^{2}\left(M_{Z}\right)>m_{H_{u}}^{2}\left(M_{Z}\right)$. From eq. (3.9), however, one finds

$$
m_{H_{d}}^{2}\left(M_{\mu}\right)-m_{H_{u}}^{2}\left(M_{\mu}\right)=8\left(z_{H_{d}}-z_{H_{u}}\right) \tilde{m}^{2}
$$

so that at the $U(1)_{\mu}$-breaking scale we already have $m_{H_{d}}^{2}\left(M_{\mu}\right)<m_{H_{u}}^{2}\left(M_{\mu}\right)$. In addition, at large $\tan \beta$ the bottom and tau Yukawa couplings are enhanced and tend to further reduce $m_{H_{d}}^{2}\left(M_{Z}\right)$.

The collider phenomenology of this class of models depends on the nature and lifetime of the next-to-lightest supersymmetric particle (NLSP). Note that our models have automatic conservation of $R$-parity, which can be defined as (recall that we are using the normalization $z_{S}=4$ )

$$
R=(-1)^{3\left[z-6 y\left(z_{Q}-1\right)\right]+2 s},
$$

where $y$ and $z$ stand for the hypercharge and $U(1)_{\mu}$ charge of a particle, and $s$ is its spin. Therefore, the NLSP can only decay to its superpartner plus a gravitino $\tilde{G}$.

First we discuss the mass spectrum, in order to determine which particles are potential NLSP candidates. Below the scale $M_{\mu}$ there are 6 neutralinos, for which we choose the basis $\left\{\tilde{B}, \tilde{W}_{3}, \tilde{H}_{d}, \tilde{H}_{u}, \tilde{\Sigma} \equiv \cos \theta \tilde{N}+\sin \theta \tilde{S}^{\prime}, \tilde{X}^{\prime}\right\}$, where $\cos ^{2} \theta \approx 2 / 3$ and

$$
\tilde{S}^{\prime}=\frac{\lambda \tilde{S}+\epsilon \tilde{X}}{\sqrt{\lambda^{2}+\epsilon^{2}}}, \quad \tilde{X}^{\prime}=\frac{\lambda \tilde{X}-\epsilon \tilde{S}}{\sqrt{\lambda^{2}+\epsilon^{2}}} .
$$


Here $\tilde{N}(\tilde{X}, \tilde{S})$ denotes the fermionic component of the SM singlet superfield $N(X, S)$. The neutralino mass matrix is given by

$$
\mathcal{M}_{\tilde{\chi}^{0}}=\left(\begin{array}{cccccc}
M_{1} & 0 & -\frac{1}{2} g^{\prime} v_{d} & \frac{1}{2} g^{\prime} v_{u} & 0 & 0 \\
0 & M_{2} & \frac{1}{2} g v_{d} & -\frac{1}{2} g v_{u} & 0 & 0 \\
-\frac{1}{2} g^{\prime} v_{d} & \frac{1}{2} g v_{d} & 0 & -\mu & -\frac{1}{\sqrt{6}} \kappa v_{u} & -\frac{1}{\sqrt{2}} \kappa^{\prime} v_{u} \\
\frac{1}{2} g^{\prime} v_{u} & -\frac{1}{2} g v_{u} & -\mu & 0 & -\frac{1}{\sqrt{6}} \kappa v_{d} & -\frac{1}{\sqrt{2}} \kappa^{\prime} v_{d} \\
0 & 0 & -\frac{1}{\sqrt{6}} \kappa v_{u} & -\frac{1}{\sqrt{6}} \kappa v_{d} & 0 & 4 \tilde{m} \\
0 & 0 & -\frac{1}{\sqrt{2}} \kappa^{\prime} v_{u} & -\frac{1}{\sqrt{2}} \kappa^{\prime} v_{d} & 4 \tilde{m} & 0
\end{array}\right),
$$

where $v_{u, d}=\sqrt{2}\left\langle H_{u, d}\right\rangle$. This situation resembles the next-to-minimal supersymmetric standard model (NMSSM) 20], except that now we have not one, but two singlet states, which are degenerate to lowest order.

The neutral Higgs masses are the same as in the MSSM, with the addition of two new CP-even singlet states with masses $2 \sqrt{6} \tilde{m}$ and $2 \sqrt{2} \tilde{m}$, and two new CP-odd singlet states with masses $2 \sqrt{6} \tilde{m}$ and $2 \sqrt{3} \tilde{m}$. The mixing between these new states and the Higgses of the MSSM $\left(h^{0}, H^{0}\right.$ and $\left.A^{0}\right)$ is suppressed by the small Yukawa couplings $\kappa$ or $\kappa^{\prime}$.

In Table 5 we list sample particle spectra for model points in each of the cases represented in Table 4 . In addition to the values of the model parameters, for completeness we also give the corresponding ratios of the fundamental parameters in the Lagrangian (coupling constants). A few comments are in order at this point. As we mentioned earlier in this Section, models with $z_{H_{d}}<z_{H_{u}}(1 \mathrm{a}(\mathrm{i}), 2 \mathrm{a}(\mathrm{iii})$ and $2 \mathrm{a}(\mathrm{iv}))$ typically require the presence of the additional coupling $\kappa^{\prime}$, in which case $\tilde{m}$ is an input. Otherwise, $\tilde{m}$ is computed from eqs. (3.5) and (3.8):

$$
\tilde{m}=\sqrt{\frac{|B \Lambda|}{12}} .
$$

If $\tilde{m}$ is large, the usual hierarchy between the left-handed and the right-handed sleptons may be affected, due to the $U(1)_{\mu}$ contributions in eq. (3.9). For example, in model 1a(i), where $z_{E}>z_{L}$ and $\tilde{m}$ is sizable, we find $m_{\tilde{e}_{R}}>m_{\tilde{e}_{L}}$, contrary to the prediction of the minimal models [11, 12]. In principle, this inverse slepton mass hierarchy is also possible for models $2 \mathrm{a}$ (iii) and $2 \mathrm{a}$ (iv). This contribution, however, is not important for the squarks, where the SM gauge-mediated contributions dominate. We also find that the $\mu$ parameter is typically larger than in the minimal gauge-mediated models, due to the negative $U(1)_{\mu}$ contributions to $m_{H_{u}}^{2}$. Note the presence of the two extra degenerate neutralinos in the spectrum. However, because of their very small couplings, their impact 


\begin{tabular}{||c||c|c|c|c|c||}
\hline \hline \multicolumn{1}{||c||}{ Particle } & \multicolumn{5}{c||}{ Models } \\
\cline { 2 - 6 } & $1 \mathrm{a}(\mathrm{i})$ & $2 \mathrm{a}(\mathrm{i})$ & $2 \mathrm{a}(\mathrm{ii})$ & $2 \mathrm{a}(\mathrm{iii})$ & $2 \mathrm{a}(\mathrm{iv})$ \\
\hline \hline$\tilde{\chi}_{1}^{0}$ & 130.8 & 164 & 81 & 120 & 120 \\
\hline$\tilde{\chi}_{2}^{0}$ & 202 & 268 & 134 & 120 & 120 \\
\hline$\tilde{\chi}_{3}^{0}$ & 400 & 724 & 507 & 126.5 & 161 \\
\hline$\tilde{\chi}_{4}^{0}$ & 400 & 724 & 509 & 201 & 258 \\
\hline$\tilde{\chi}_{5}^{0}$ & 575 & 793 & 544 & 383 & 451 \\
\hline$\tilde{\chi}_{6}^{0}$ & 580 & 797 & 544 & 401 & 465 \\
\hline \hline$\tilde{\chi}_{1}^{+}$ & 131.0 & 268 & 134 & 200 & 258 \\
\hline$\tilde{\chi}_{2}^{+}$ & 581 & 798 & 513 & 401 & 466 \\
\hline \hline$\tilde{e}_{R}$ & 253 & 262 & 248 & 131 & 155 \\
\hline$\tilde{e}_{L}$ & 247 & 427 & 272 & 217 & 266 \\
\hline$\tilde{\tau}_{1}$ & 166 & 147 & 216 & 125.2 & 125 \\
\hline$\tilde{\tau}_{2}$ & 312 & 478 & 300 & 220 & 277 \\
\hline \hline$\tilde{g}^{\prime}$ & 1126 & 1141 & 615 & 924 & 1134 \\
\hline$\tilde{t}_{1}$ & 984 & 1045 & 589 & 795 & 979 \\
\hline$\tilde{u}_{R}$ & 1074 & 1112 & 610 & 866 & 1061 \\
\hline \hline$h^{0}$ & 114 & 113 & 109 & 111 & 114 \\
\hline$H^{0}$ & 379 & 487 & 177 & 339 & 454 \\
\hline \hline$M[\mathrm{TeV}]$ & 500 & 200 & 100 & 200 & 200 \\
\hline$\Lambda[\mathrm{TeV}]$ & 50 & 50 & 25 & 40 & 50 \\
\hline$M_{\mu}[\mathrm{TeV}]$ & 10,000 & 1,000 & 10,000 & 5,000 & 2,000 \\
\hline $\tan \beta$ & 35 & 60 & 25 & 10 & 25 \\
\hline$\mu\left(M_{\mu}\right)$ & 602 & 862 & -537 & 387 & 460 \\
\hline$\tilde{m}$ & 100 & 182 & 156 & 30 & 30 \\
\hline \hline$\kappa / \lambda$ & 1.74 & 1.37 & 1.14 & 3.72 & 4.43 \\
\hline$\epsilon / \lambda$ & 0.0069 & 0.0126 & 0.0188 & 0.0026 & 0.002 \\
\hline$\kappa^{\prime} / \lambda$ & 0.0796 & - & - & 1.545 & 0.713 \\
\hline \hline & & & & & \\
\hline
\end{tabular}

Table 5: Sample particle spectra for the models in Table 1. 
on phenomenology is negligible, unless one of them is the NLSP - see the examples for models $2 \mathrm{a}(\mathrm{iii})$ and $2 \mathrm{a}(\mathrm{iv})$.

Table 5 rather nicely illustrates all potential NLSP candidates in our models:

1. The lightest neutralino, which is mostly wino-like: $\tilde{\chi}_{1}^{0} \sim \tilde{W}_{3}^{0}$. This situation may arise in any one of the models with $\Delta \beta_{2}=1$, where at the weak scale we find the reversed gaugino mass hierarchy $M_{3}: M_{1}: M_{2} \sim 9: 1.5: 1$. Since $M_{2}$ is also the soft mass of the wino-like chargino, one faces the dilemma of deciding which one is actually the NLSP: the chargino or the neutralino. (Quite recently, the case of $M_{2}<M_{1}$ was discussed in the framework of supergravity-mediated (SUGRA) models, where the soft masses arise through the super-conformal anomaly [21, 22.) At tree-level, one can expand the lightest chargino and neutralino mass eigenvalues in terms of $1 /|\mu|$ :

$$
\begin{aligned}
& m_{\tilde{\chi}_{1}^{+}}=M_{2}-\frac{M_{W}^{2}}{\mu} s_{2 \beta}-\frac{M_{W}^{2}}{\mu^{2}} M_{2}+\mathcal{O}\left(\frac{1}{\mu^{3}}\right) \\
& m_{\tilde{\chi}_{1}^{0}}=M_{2}-\frac{M_{W}^{2}}{\mu} s_{2 \beta}-\frac{M_{W}^{2}}{\mu^{2}} M_{2}-\frac{M_{W}^{2}}{\mu^{2}} \frac{M_{W}^{2}}{M_{1}-M_{2}} t_{W}^{2} s_{2 \beta}^{2}+\mathcal{O}\left(\frac{1}{\mu^{3}}\right),
\end{aligned}
$$

where $t_{W} \equiv \tan \theta_{W}$ and $s_{2 \beta} \equiv \sin 2 \beta$. We find that the mass splitting occurs only at order $1 /\left|\mu^{2}\right|$ and the chargino is always heavier at tree-level:

$$
\Delta m_{\chi} \equiv m_{\tilde{\chi}_{1}^{+}}-m_{\tilde{\chi}_{1}^{0}}=\frac{M_{W}^{2}}{\mu^{2}} \frac{M_{W}^{2}}{M_{1}-M_{2}} t_{W}^{2} s_{2 \beta}^{2}+\mathcal{O}\left(\frac{1}{\mu^{3}}\right) .
$$

Notice the additional suppression at large $\tan \beta$ due to the factor $\sin ^{2} 2 \beta \sim 4 / \tan ^{2} \beta$, in which case the next order terms may be numerically important as well. Typical values of the parameters result in a mass splitting $\Delta m_{\chi}$ in the $\mathrm{MeV}$ range. In any case, we see that in order to correctly determine the nature of the NLSP, it is necessary to account for the one-loop gaugino mass corrections [23]. Including the full one-loop mass corrections to the chargino and neutralino matrices [24], we find that the neutralino is indeed the NLSP, and the mass splitting is in fact much larger than predicted by eq. (3.20). We illustrate this result in Fig. 11. Even though the chargino and neutralino mass corrections themselves are dominated by the squark and Higgs loops, we have checked that the renormalization of the mass splitting is due almost entirely to the gauge boson loops. For small chargino or neutralino

\footnotetext{
${ }^{3}$ Our result for both the chargino and neutralino differs from that of Refs. [21, 22].
} 


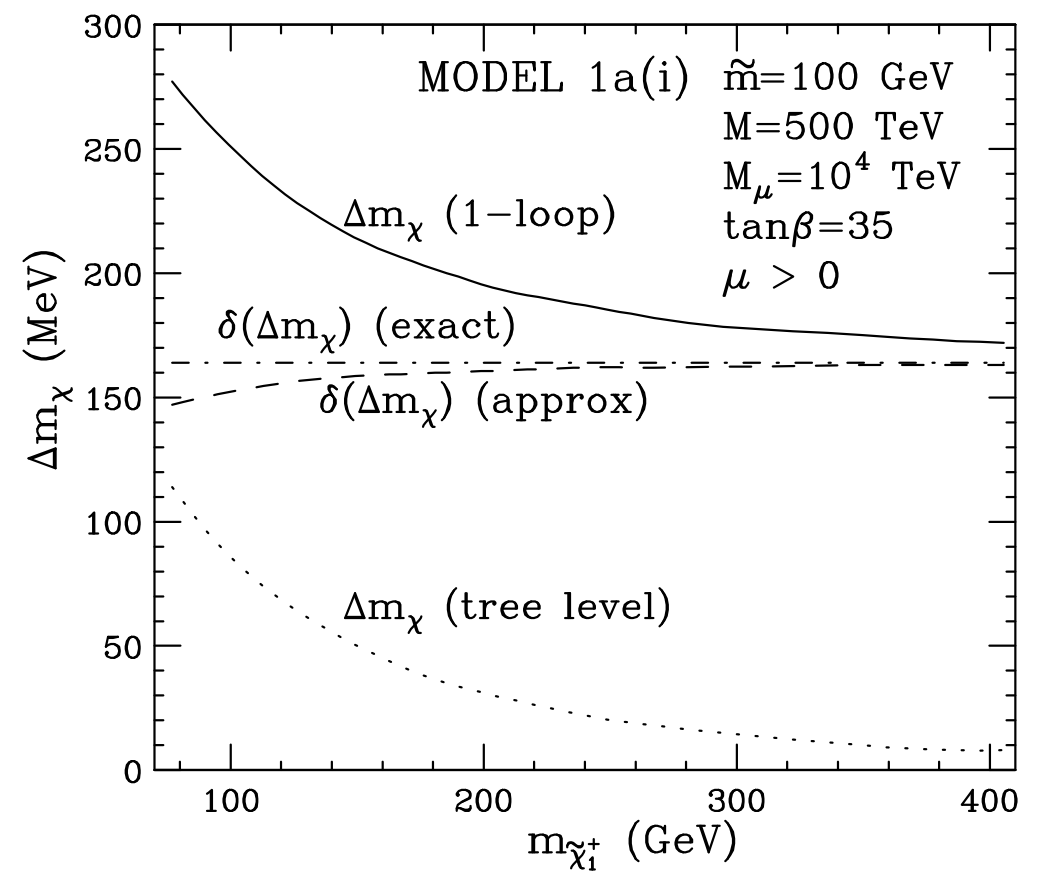

Figure 1: The mass splitting $\Delta m_{\chi} \equiv m_{\tilde{\chi}_{1}^{+}}-m_{\tilde{\chi}_{1}^{0}}$ at tree-level (dotted) and one-loop (solid), versus the chargino mass $m_{\tilde{\chi}_{1}^{+}}$, which is varied by varying $\Lambda$. The dot-dashed line represents the exact one-loop correction $\delta \Delta m_{\chi}$, and the dashed line is the result from the approximation in eq. (3.21).

mixing, and keeping only the gauge boson contributions, we can derive the following approximate formula for the one-loop correction to $\Delta m_{\chi}$ :

$$
\begin{aligned}
\delta \Delta m_{\chi} & \equiv \Delta m_{\chi}^{1-\text { loop }}-\Delta m_{\chi}^{\text {tree }} \\
& =\frac{g^{2}}{8 \pi^{2}}\left[2 c_{W}^{2} B_{0}\left(M_{2}, M_{2}, M_{Z}\right)+2 s_{W}^{2} B_{0}\left(M_{2}, M_{2}, 0\right)-2 B_{0}\left(M_{2}, M_{2}, M_{W}\right)\right. \\
& \left.-c_{W}^{2} B_{1}\left(M_{2}, M_{2}, M_{Z}\right)-s_{W}^{2} B_{1}\left(M_{2}, M_{2}, 0\right)+B_{1}\left(M_{2}, M_{2}, M_{W}\right)\right] M_{2},(3.21)
\end{aligned}
$$

with the functions $B_{0}$ and $B_{1}$ defined as in Appendix B of Ref. [24]. Notice that this correction is purely finite and cannot be accounted for in a leading-log decoupling scheme. Since the dominant effect is from the gauge boson loops only, the result (3.21) is quite model-independent and will apply for the supergravity-mediated models discussed in Refs. [21, 22] as well.

Since the lightest chargino and neutralino are so degenerate, the decay length $L_{\tilde{\chi}}$ 
for the decay $\tilde{\chi}_{1}^{+} \rightarrow \tilde{\chi}_{1}^{0}+X$ could be macroscopic [25]:

$$
L_{\tilde{\chi}}=\left(\frac{1 G e V}{\Delta m_{\chi}}\right)^{5}\left(\frac{E^{2}}{m_{\tilde{\chi}}^{2}}-1\right)^{1 / 2} \times 100 \mu m .
$$

For typical mass splittings $\Delta m_{\chi} \sim 200 \mathrm{MeV}$ (see Fig. 1), $L_{\tilde{\chi}}$ is on the order of tens of centimeters. In that case, the lightest chargino and neutralino may act as co-NLSP's, if the decays to gravitinos are faster.

2. In any one of our models, the limit $\tilde{m} \rightarrow 0$ gives rise to a neutralino NLSP, which is a mixture of $\tilde{\Sigma}$ and $\tilde{X}^{\prime}$. We see such examples in Table 5 for models 2 a(iii) and $2 \mathrm{a}(\mathrm{iv})$, but we find that small $\tilde{m}$ is possible for all other models as well.

3. In all models with $\Delta \beta_{2}=2,3$ or 4 we find that $M_{1}<M_{2}$, so that the lightest neutralino is mostly $\tilde{B}$, as in the conventional SUGRA or minimal gauge-mediated models. For either moderate values of $\tan \beta$ or rather large values of $\tilde{m}$, it also turns out to be the NLSP - see e.g. model 2a(ii) in Table 5. The phenomenology of similar gauge-mediated models, albeit with a somewhat different gaugino mass splitting, has been extensively discussed in the literature [26].

4. The lightest tau slepton $\tilde{\tau}_{1}$ can be the NLSP if $\tan \beta$ is significant and $\tilde{m}$ is not too large, e.g. in model $2 \mathrm{a}(\mathrm{i})$ of Table 5. This case is not much different from the minimal gauge-mediated models with a stau NLSP and has been studied previously [27, 28] for both stable and promptly decaying staus.

The other important factor in the discussion of the typical collider signatures of our models is the value of the intrinsic SUSY breaking scale $E_{\mathrm{vac}}$, which determines the decay length $L_{\mathrm{NLSP}}$ of the corresponding NLSP:

$$
L_{\mathrm{NLSP}} \sim 130\left(\frac{100 \mathrm{GeV}}{m_{\mathrm{NLSP}}}\right)^{5}\left(\frac{E_{\mathrm{vac}}}{100 \mathrm{TeV}}\right)^{4} \mu m,
$$

with $E_{\mathrm{vac}}^{4}$ being the vacuum energy density. The value of $E_{\mathrm{vac}}$ in our models is given by [6]

$$
E_{\mathrm{vac}} \gtrsim \mathcal{O}(1)\left(\frac{4 \pi}{g_{\mu}}\right) \sqrt{F_{X}} \gtrsim \mathcal{O}(1) \times 200 \mathrm{TeV}
$$

We see that for $E_{\mathrm{vac}}$ close to the lower limit $\left(\sim 10^{5} \mathrm{GeV}\right), L_{\mathrm{NLSP}}$ could be microscopic and unlike most known models of gauge-mediated SUSY breaking, prompt decays of the NLSP are possible. 
In the rest of this section we shall concentrate on the first two NLSP options, since they are unique features of our models. Prompt decays of the $\tilde{W}$-like chargino and $\tilde{W}_{3}$-like neutralino co-NLSP's in the models with $\Delta \beta_{2}=1$ lead to signatures which have never before been discussed as possible SUSY discovery modes, so we devote the next subsection 3.2 to this case. Later in subsection 3.3 we discuss the phenomenology of the singletino NLSP scenario, which resembles somewhat that of a gauge-mediated NMSSM. Finally, we conclude this Section with comments on the more standard cases of $\tilde{B}$-like neutralino or stau NLSP.

\section{2 $S U(2)$-neutralino NLSP}

Type 1 models (see Table 1) have the generic prediction $M_{2}<M_{1}<M_{3}$ and the lightest neutralino is mostly $\tilde{W}_{3}$. As shown in the previous subsection, the lightest neutralino and the lightest chargino in this case are degenerate enough so that they can act as co-NLSP's. The typical experimental signatures therefore depend on which charginoneutralino combinations are mostly being produced. At the Tevatron, the dominant production processes are $p \bar{p} \rightarrow \tilde{\chi}_{1}^{+} \tilde{\chi}_{1}^{-}$and $p \bar{p} \rightarrow \tilde{\chi}_{1}^{ \pm} \tilde{\chi}_{1}^{0}$, which are roughly of the same order, while $p \bar{p} \rightarrow \tilde{\chi}_{1}^{0} \tilde{\chi}_{1}^{0}$ is much smaller. In the rest of this subsection, we shall therefore only consider the fate of a $\tilde{\chi}_{1}^{+} \tilde{\chi}_{1}^{-}$or a $\tilde{\chi}_{1}^{ \pm} \tilde{\chi}_{1}^{0}$ gaugino pair.

If the SUSY breaking scale $E_{\mathrm{vac}}$ is high, the decays of both the chargino and the neutralino to gravitinos will happen outside the detector and the signatures are similar to those discussed in Ref. [25, 21, 22] for supergravity-mediated models. In this case the chargino will have time to decay to a neutralino first. However, it is rather unlikely that the chargino will make it out to the muon chambers - we saw that the one-loop corrections tend to increase the $\tilde{\chi}_{1}^{ \pm}-\tilde{\chi}_{1}^{0}$ mass splitting and the chargino decay will probably occur within a meter or so from the primary vertex, thus evading existing limits from heavy charged stable particle searches [29]. It will therefore look like a tau and will be rather difficult to identify [21]. Because of the small chargino-neutralino mass splitting, the lepton from the $\tilde{\chi}_{1}^{ \pm} \rightarrow \tilde{\chi}_{1}^{0} l^{ \pm} \nu$ decay will be very soft and cannot be used to tag the chargino decay. Note also that this mass degeneracy renders the current LEP limits on the chargino mass inapplicable.

As in any model with a rather low SUSY breaking scale, decays of the NLSP to $\tilde{G}$ provide information about the hidden (or messenger) sector via $L_{\mathrm{NLSP}}$. If it is finite ( $\gtrsim 1$ $\mathrm{mm}$ ) and the NLSP's ( $\tilde{\chi}_{1}^{ \pm}$or $\left.\tilde{\chi}_{1}^{0}\right)$ decay to gravitinos inside the detector, this will give rise to events with displaced vertices (kinks in the charged tracks), photons with finite impact 
parameters or originating from the outer hadronic calorimeter [30]. A recent CDF search for long-lived $Z$-parents [31] is not sensitive enough to place a limit on the neutralino mass in this case. Because of the phase space suppression, the branching ratio $B R\left(\tilde{\chi}_{1}^{0} \rightarrow Z \tilde{G}\right)$ begins to dominate over $B R\left(\tilde{\chi}_{1}^{0} \rightarrow \gamma \tilde{G}\right)$ only for neutralino masses $m_{\tilde{\chi}_{1}^{0}} \gtrsim 130 \mathrm{GeV}$, where the production cross-section falls below the Run I sensivity.

Finally, if the SUSY breaking scale $E_{\text {vac }} \sim 10^{5} \mathrm{GeV}$, the chargino and neutralino coNLSP's may decay promptly to gravitinos, creating events with real $W$ 's, $Z$ 's or photons and missing (transverse) energy. Since the signatures for $\tilde{\chi}_{1}^{+} \tilde{\chi}_{1}^{-}$and $\tilde{\chi}_{1}^{ \pm} \tilde{\chi}_{1}^{0}$ production are different, we shall discuss each case in turn.

For chargino pair production with subsequent prompt decays to gravitinos, the possible final state signatures are $l^{+} l^{-} E_{T}, \operatorname{lj} j E_{T}$ and $j j j j E_{T}$, with branching ratios $6 \%, 38 \%$ and $56 \%$, correspondingly. The two leptonic signatures suffer from large irreducible $W$-pair and $t-\bar{t}$ backgrounds, although the latter one may be somewhat suppressed via a $b$-jet veto. These two channels have been previously considered as possible Standard Model Higgs search modes at both the Tevatron and LHC [32, 33, 34, since for $m_{h}>140$ $\mathrm{GeV}$ the branching ratio $B R\left(h \rightarrow W^{+} W^{-}\right)$starts to dominate. The result is that this signal will be rather difficult to observe at the Tevatron, and a $3 \sigma$ discovery is only possible with Run III integrated luminosities $L_{\text {int }} \sim 30 \mathrm{fb}^{-1}$ [34]. For a certain range of chargino masses, we can immediately adapt this result to our case. For Higgs masses in the range $140-180 \mathrm{GeV}$, the cross-section for $W$-pair production via single Higgs is $\sigma_{h}\left(g g \rightarrow h^{0} \rightarrow W W\right) \sim 0.2-0.4 \mathrm{pb}$. For chargino masses in the range $130-150 \mathrm{GeV}$, the signal cross-section $\sigma_{\tilde{\chi}}\left(p \bar{p} \rightarrow \chi^{+} \chi^{-}+X\right)$ is of the same order, so we conclude that only Run III at the Tevatron may possibly have some sensitivity beyond LEP-II in those two channels. For smaller chargino masses, the Tevatron reach is better and a signal may be observed in the very early stages of Run III. In the most optimistic scenario, where the chargino mass is just beyond the projected LEP-II limit $\left(m_{\tilde{\chi}_{1}^{+}} \sim 100 \mathrm{GeV}\right), \sigma_{\tilde{\chi}} \sim 1.2 \mathrm{pb}$ and can be observed even in Run II.

The other possible signal of $\tilde{\chi}_{1}^{+} \tilde{\chi}_{1}^{-} \rightarrow W^{+} W^{-} \tilde{G} \tilde{G}$ is the multijet channel, which has rather small SM physical backgrounds (the $t-\bar{t}$ background can be suppressed with a lepton veto). The single Higgs production analogy now does not work, because of the $E_{T}$ requirement. The dominant background is from QCD multijet production and jet energy mismeasurement, which is why a detailed Monte Carlo study with a very realistic detector simulation is necessary in order to estimate the reach in this channel. In addition to a hard $\mathbb{E}_{T}$ cut, one may also make use of the fact that two different jet pairs should 
reconstruct close to the $W$ mass.

We now turn to the signatures arising in the $\tilde{\chi}_{1}^{ \pm} \tilde{\chi}_{1}^{0}$ case, where we have to factor in the branching ratios of the neutralino to a $Z$ or a photon. For relatively light neutralinos, it is best to study signatures where the neutralino decays to a photon and a gravitino. First, for $m_{\tilde{\chi}_{1}^{0}} \lesssim M_{Z}$, this is the dominant decay mode $(\sim 100 \%)$ anyways. Second, even when $m_{\tilde{\chi}_{1}^{0}}>M_{Z}$ and the decay to $Z$ dominates, the $B R\left(\tilde{\chi}_{1}^{0} \rightarrow \gamma \tilde{G}\right)$ is never below $\sim 20 \%$, which is still better than the leptonic branchings of the $Z$ 's (the channels with hadronic $Z$ 's have larger backgrounds). We conclude that the most promising clean signature in this case is $l^{ \pm} \gamma \mathbb{E}_{T}$. The only physical background process is $W \gamma$, which is rather rare, so the typical backgrounds will involve photon/lepton misidentification and/or $E_{T}$ mismeasurement. Note that in contrast to the minimal gauge-mediated models, our type 1 models are not associated with any di-photon signatures 35], because the neutralino pair-production cross-sections are suppressed, while the chargino decay does not yield a photon.

Finally, there is a variety of possible signatures, if we consider prompt neutralino decays to $Z$ 's. We shall concentrate on the following channels: $l^{+} l^{-} l^{ \pm} E_{T} ; l^{+} l^{-} j j E_{T}$; $l^{ \pm} j j E_{T}$ and $j j j j E_{T}$, since $l^{ \pm} E_{T}$ and $j j E_{T}$ have too large a background to be even considered.

The clean trilepton signature has irreducible background from $W Z$ and in addition one takes a hit from the $Z$ branching ratio of the neutralino, so it is rather unlikely that an excess of such events will be seen in any of the future Tevatron runs. Unlike the classic SUSY trilepton signature [36], one cannot use an invariant dilepton mass cut to beat down the $W Z$ background. The case of the $l^{ \pm} j j E_{T}$ is even worse: it has large irreducible backgrounds from both $W Z$ and $t \bar{t}$.

The dilepton plus jets signature $l^{+} l^{-} j j \quad E_{T}$ looks somewhat promising. It was used to search for cascade decays of gluinos and/or squarks [37]. The difference now is that the leptons are coming from a $Z$-decay, so the invariant dilepton mass cut is exactly the opposite of what is used in the conventional SUSY search. The dominant physical backgrounds then would be $Z j j \rightarrow \tau^{+} \tau^{-} j j \rightarrow l^{+} l^{-} j j E_{T}$, and to some extent $t-\bar{t}$. Both of them can be significantly reduced by requiring that the jet pair reconstructs the $W$ mass.

The 4-jet plus $E_{T}$ signature was already discussed above for the case of hadronically decaying $W^{\prime}$ 's in chargino pair-production, the difference now is that the two jet pairs should reconstruct the $W$ and $Z$ mass, correspondingly, so that one should use a more relaxed cut, e.g., $70 \mathrm{GeV}<m_{j j}<100 \mathrm{GeV}$. 


\subsection{Singletino NLSP}

In the limit of small $\tilde{m}$ the two lightest neutralinos will be rather degenerate and have significant "singletino" components from $\tilde{\Sigma}$ and $\tilde{X}^{\prime}$. Since their masses are of order $4 \tilde{m}$, while the mass of the lightest scalar singlet $H_{S}$ is only $2 \sqrt{2} \tilde{m}$, the "singletino"-like NLSP will always decay as $\tilde{\chi}_{1}^{0} \rightarrow H_{S} \tilde{G}$. $H_{S}$ will subsequently decay to $b-\bar{b}$, due to the small $S-\left\{H_{u}, H_{d}\right\}$ mixing. If the singletino decays some distance away from the primary vertex, this will give rise to rather spectacular signatures with displaced $b$-jets. The case when the singletinos decay promptly resembles that of the minimal gauge-mediated models with a short-lived higgsino NLSP [38], heavier than the light Higgs $h^{0}$. The difference now is that the jet pairs should reconstruct the mass of the singlet Higgs $H_{S}$ rather than $h^{0}$. Note that the LEP limits on the Higgs mass do not directly apply to $H_{S}$.

If the singletinos decay outside the detector, the typical signatures depend on the nature of the next-to-next-to-lightest supersymmetric particle (NNLSP). Because of the small couplings of the 'singletinos', all supersymmetric particles will first decay to the NNLSP. For the models from Table 5, the NNLSP is typically $\tilde{\tau}_{1}$, which can be understood as follows. The singletino NLSP case arises for small values of $\tilde{m}$, when the $U(1)_{\mu}$ contributions to the scalar masses are also small. Then, the supersymmetric mass spectrum in any of our models resembles that of a minimal gauge-mediated model, with the corresponding number and type of messenger representations. Thus we can immediately adapt the NLSP analysis in the minimal gauge mediated models to the question of the NNLSP in our models. The balance between the masses of the two main NNLSP candidates: stau and $\tilde{B}$-like neutralino, is for the most part determined by the value of the messenger multiplicity factor $\Delta \beta_{1}$, since $m_{\tilde{\tau}_{1}} \sim \sqrt{\Delta \beta_{1}}$, while $m_{\tilde{B}} \sim \Delta \beta_{1}$. In models of type 1 and 2, $\Delta \beta_{1}$ is large, and the stau is lighter than the bino throughout most of the parameter space. One should keep in mind though that in models 1 the stau mass should be compared to the $\tilde{W}_{3}$-like neutralino mass instead, so that cases with $m_{\tilde{\chi}_{1}^{0}}<m_{\tilde{W}_{3}}<m_{\tilde{\tau}_{1}}<m_{\tilde{B}}$ are certainly possible. Note that at low enough values of $\tan \beta$ and $\Lambda$ one can reach a situation where $m_{\tilde{\chi}_{3}^{0}}-m_{\tilde{\tau}_{1}}<m_{\tau}$, so that the stau and the bino are in fact co-NNLSP's. Such an example is shown in Table 5 for model 2a(iii). Next, for $\Delta \beta_{1}=2$ (models of type 4), one typically finds $m_{\tilde{\chi}_{3}^{0}}<m_{\tilde{\tau}_{1}}$. Finally, for $\Delta \beta_{1}=3$ (models of type 3 ), one finds cases with either stau or bino NNLSP.

Turning on to the collider phenomenology of models with stable singletino NLSP, we

\footnotetext{
${ }^{4}$ We do not count the second singletino.
} 


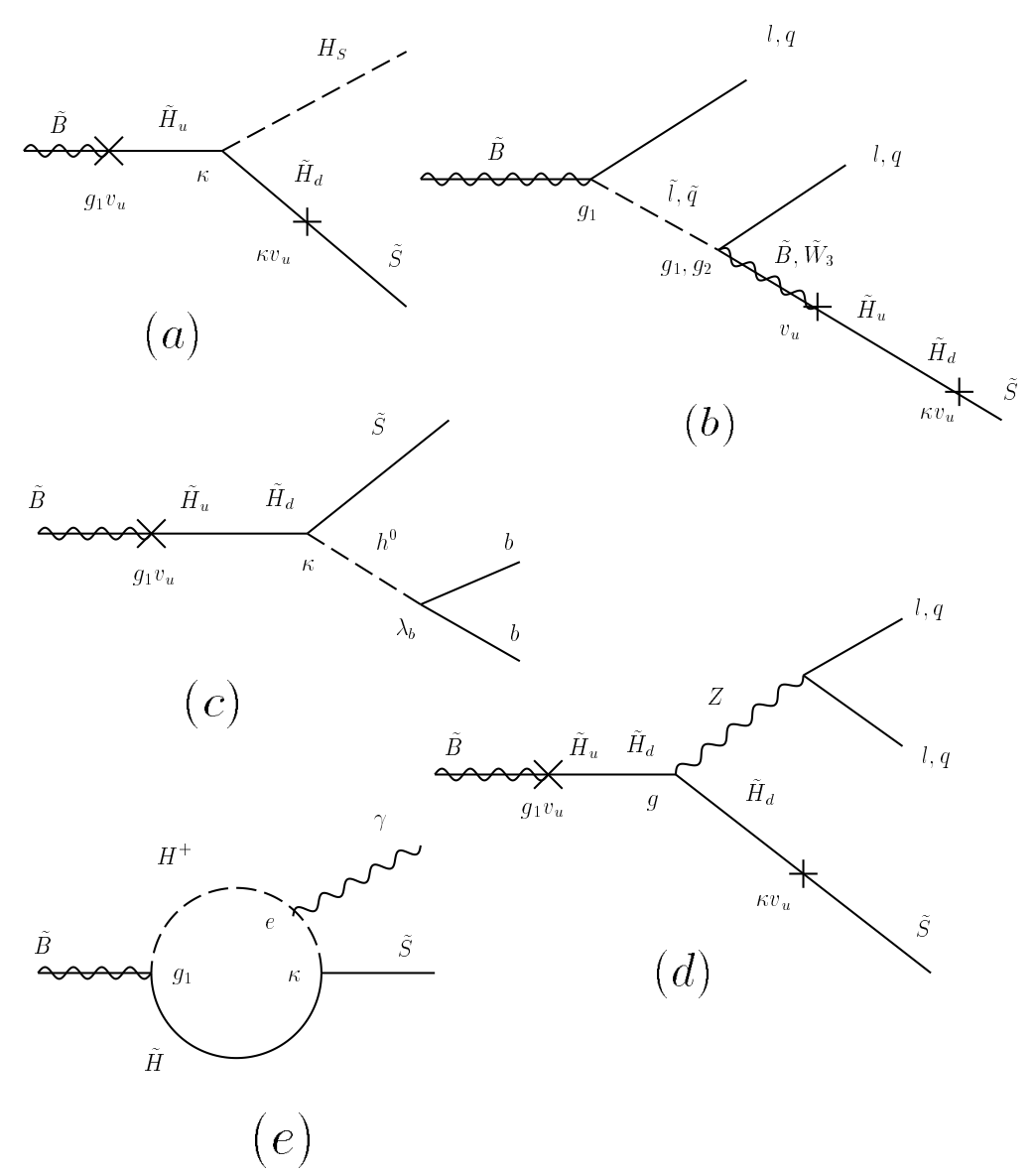

Figure 2: Sample diagrams for the possible decay modes of $\tilde{\chi}_{3}^{0}$ to the singletino NLSP $\tilde{\chi}_{1}^{0}$.

first discuss the stau NNLSP case. In principle, each SUSY event will contain at least two taus from the $\tilde{\tau}_{1} \rightarrow \tilde{\chi}_{1}^{0} \tau$ decays. Their $p_{T}$ spectrum is determined by the mass difference $m_{\tilde{\tau}_{1}}-m_{\tilde{\chi}_{1}^{0}}$, and may be quite soft - see the $2 \mathrm{a}$ (iii) example in Table 5. To make matters worse, the tau jets and especially the leptons from the tau decays will be even softer, presenting serious triggering and identification problems.

The distinctive collider signature in case of a neutralino co-NNLSP depends on which is the dominant decay of $\tilde{\chi}_{3}^{0}$ to the singletino NLSP. There are three possibilities:

1. The two-body decay $\tilde{\chi}_{3}^{0} \rightarrow \tilde{\chi}_{1}^{0} H_{S}$ may be open for $\tilde{m} \lesssim M_{1} / 6.8$. This decay proceeds via the diagram shown in Fig. \&(a) and one can see that the rate is suppressed by four powers of $\kappa$ or $\kappa^{\prime}$, as well as the gaugino-higgsino mixing.

2. For values of $\tilde{m} \gtrsim M_{1} / 6.8$, the tree-level two body decays of $\tilde{\chi}_{3}^{0}$ are closed and the three-body decays via the diagrams in Fig. 2(b)-(d) are possible. They are typically suppressed by only two powers of $\kappa$ or $\kappa^{\prime}$, in addition to the gaugino-higgsino mixing. 
3. The radiative decay $\tilde{\chi}_{3}^{0} \rightarrow \tilde{\chi}_{1}^{0} \gamma$ (Fig. $\mathbb{Q}(\mathrm{e})$ ) is also possible. It becomes important when the $\tilde{B}$ and $\tilde{\Sigma}\left(\tilde{X}^{\prime}\right)$ masses are very close and the three-body decays are suppressed. Unlike the previous decays, this mode has no gaugino-higgsino mixing suppression.

The relative importance of these three modes will depend on the particular values of the model parameters [39]. A more quantitative analysis will have to take into account the correct singletino-gaugino-higgsino mixing as well as the singlet-Higgs mixing.

We conclude this Section with some comments on the more conventional cases of $\tilde{B}$ or stau NLSP. For the most part, they are very similar to the corresponding minimal gauge-mediated models, and the results from previous phenomenological analyses hold [26, 27, 28]. However, there are two differences. First, the predicted gaugino mass ratios are different. This is important e.g. in the case of a 'stable' Bino-NLSP, since the $p_{T}$ distributions of the $\tilde{\chi}_{1}^{+}$and $\tilde{\chi}_{2}^{0}$ decay products will be affected. For a given $\tilde{\chi}_{1}^{+}$mass (i.e. signal cross-section), we would expect softer (harder) $p_{T}$ spectra for models 2 (3-4), which will have an impact on the cuts optimization. Second, in the minimal gauge-mediated models, for $\mu>0$, large values of $\tan \beta$ are typically excluded because the light stau is below the experimental limit. In our models, with the possibility of the stau mass to receive additional positive contributions from the $U(1)_{\mu}$ D-term, we find that the large $\tan \beta$ part of the parameter space for $\mu>0$ can be extended up to $\tan \beta \sim 70$, where either $m_{A}^{2}<0$ or the tau Yukawa coupling diverges below the Planck scale (the bottom Yukawa coupling is less of a problem, since for $\mu>0$ it is reduced by the SUSY threshold corrections).

\section{Discussion and Conclusions}

In this section we discuss how robust our model selection assumptions are, we summarize the phenomenological signatures, and we comment on the general features of the models. We start with a list the most notable constraints on model-building and we comment on their necessity:

- Viability of the models even if any global symmetry (which is not an accidental result of the gauge structure) is badly violated by Planck scale physics.

\footnotetext{
${ }^{5}$ The exact numerical bound depends on $\Lambda$ and the number of messenger pairs.
} 
To this end, the models have to be chiral (i.e. there are no gauge invariant mass terms), and generic (i.e. there are no gauge invariant and supersymmetric dimension-four operators with exceedingly small coefficients in the Lagrangian; in practice we may allow dimensionless couplings as small as the Yukawa coupling of the electron). Hence, the $\mu$-term is induced only after a gauge symmetry is spontaneously broken, while baryon number conservation is a consequence of the gauge symmetry.

This constraint is a major motivation for the model-building effort presented in Section 2. So far there is no rigorous proof that the global symmetries of the MSSM are violated by Planck scale physics if they are not protected by gauge invariance. However, this may be the case, and therefore it is important to search for extensions of the MSSM which remain viable independent of the quantum gravitational effects.

- The minimality of the gauge group: $\mathrm{SM} \times U(1)_{\mu} \times \mathrm{DSB}$.

The gauge group has to include the standard $S U(3)_{C} \times S U(2)_{W} \times U(1)_{Y}$ and some DSB gauge group responsible for breaking supersymmetry. It is remarkable that the addition of the $U(1)_{\mu}$ gauge group is sufficient to prevent the potentially dangerous Planck scale effects and to communicate supersymmetry breaking to the MSSM fields. In principle, the $U(1)_{\mu}$ may be replaced by a larger gauge group, but in that case it would be harder to cancel the mixed gauge anomalies.

- The cancellation of the mixed $\mathrm{SM} \times U(1)_{\mu}$ anomalies of the MSSM fields by the messenger sector, and of the remnant $U(1)_{\mu}$ and $U(1)_{\mu}^{3}$ anomalies by the DSB sector. These are nice features of our models because the existence of the three sectors (MSSM, messenger and DSB) is required by the mathematical consistency of the theory. This is to be contrasted with the original gauge mediated supersymmetry breaking models [11, 12] where the three sectors are introduced ad-hoc, for phenomenological reasons.

- The quark and lepton masses are generated by the Yukawa couplings to the Higgs vevs.

This assumption is convenient but does not help in explaining the pattern of observed quark and lepton masses. If one allows only some of the fermions to couple to the Higgs doublets, while inducing the other quark and lepton masses using a Frogatt-Nielsen sector, higher dimensional operators, or other mechanism, then the 
$U(1)_{\mu}$ charge assignment can be more general so that completely different models may be constructed. We will not elaborate further this possibility.

- The neutrinos have masses and the mixings involve all three generations.

As suggested by the solar, atmospheric, and accelerator neutrino experiments, we have allowed the most general Yukawa couplings of the neutrinos to the Higgs. This constraint can be relaxed, for example if there are enough sterile neutrinos. In that case the lepton $U(1)_{\mu}$ charges no longer need to be generational independent. We also assume that the Majorana masses for the right-handed neutrinos come from $\langle N\rangle$, which results in automatic $R$-parity conservation. If right-handed neutrinos obtain their masses from $\langle S\rangle, R$-parity violating operators which violate lepton number will exist and their couplings have to be quite small. Of course, even a small $R$-parity violating coupling can allow the NLSP to decay to jets and/or leptons instead, thus changing the typical collider signatures correspondingly.

- The $U(1)_{\mu}$ charges of the quarks and leptons are positive.

This constraint is sufficient to ensure that the squarks and sleptons do not acquire vevs, but is not necessary. There could be regions in the parameter space where the positive contributions to the squark and slepton squared-masses from standard gauge mediation dominate over the $U(1)_{\mu} D$ term contribution. In that case negative $U(1)_{\mu}$ charges for the quarks and leptons may be allowed. Squark and gluino masses are insensitive to this contribution, but it may affect the slepton spectrum and the question of NLSP. However, even restricting ourselves to models with positive $U(1)_{\mu}$ charges for quarks and leptons, we have found examples which exhaust all possible NLSP canditates, so considering negative charges will not give us anything new as far as phenomenology is concerned.

- The set of SM singlet superfields from the messenger sector is minimal.

It is possible to find various ways of extending the messenger sector. For example, there can be more $X$ fields, with non-zero vevs for the scalar and $F$-components, which would result in a more general squark and slepton spectrum. However, with more singlets, it is harder to find a viable minimum.

- The $U(1)_{\mu}$ charges are reasonably simple.

This assumption is necessary only if one wants to be able to embed $U(1)_{\mu}$ in a ("reasonably simple") non-Abelian gauge group. 
- There are no fields with fractional electric charge.

Such fields would be stable and produced in large numbers in the Early Universe, which is in disagreement with a wide range of experiments. This constraint can be avoided if the number of particles with fractional electric charge has been dramatically diluted during a period of inflation that ended at a temperature below their masses.

- The messenger fields can decay via dimension-4 operators.

Otherwise the lightest messenger is long lived and its presence at the time of nucleosynthesis is ruled out by cosmological observations. Again, this constraint can be relaxed if the Universe suffered a period of late inflation. Without this assumption, we find solutions for other classes of models as well.

- The DSB sector does not give rise to more than two composite chiral fermions charged under $U(1)_{\mu}$.

This assumption was made only for simplicity.

We point out that the phenomenology of these models is rather insensitive to some of the extensions listed above. For example, the last three assumptions itemized do not affect some of the novel phenomenological features discussed in Section 3:

1. Non-standard (yet predictable) gaugino mass ratios.

2. Light singlet fermion and/or scalar states may sometimes be in the spectrum.

3. The models allow for the intrinsic SUSY breaking scale $E_{\text {vac }}$ to be quite low, on the order of a few times $10^{5} \mathrm{GeV}$, thus allowing prompt decays of the NLSP. Note that other models with the SUSY breaking scale below $10^{6} \mathrm{GeV}$ are known [40], but their viability relies on assumptions about noncalculable strong dynamics

4. In certain cases we find new NLSP candidates: $\tilde{W}$-like chargino, $\tilde{W}_{3}$-like neutralino or $\tilde{S}$-like neutralino ("singletino").

It is worth emphasizing that the new SUSY discovery signatures of $W W E_{T}, W \gamma E_{T}$ and $W Z \mathbb{H}_{T}$ depend only on two assumptions: $M_{2}<M_{1}, M_{3}$ and a low SUSY breaking scale. Therefore, the importance of these signatures, which have been overlooked until now, transcends the models introduced in this paper. Even though we only discussed the 
phenomenological signatures of our models for the case of the Tevatron, it is clear that the LHC, where statistics is not an issue, will be able to definitively explore these models via the clean signatures considered in Section 3.

In conclusion, we have constructed several classes of gauge-mediated models which provide a rather complete answer to the question of SUSY-breaking and communication to the visible sector. The models allow acceptable neutrino masses, and at the same time avoid the $\mu$ problem and the difficulties with FCNC, baryon number violation and messenger dark matter.

In retrospect, our models still leave several unsolved puzzles. Most importantly, we have not attempted to explain the pattern of quark and lepton masses. Some relatively small Yukawa couplings are still needed for them and also for the $U(1)_{\mu}$ breaking sector. In addition, we have not addressed the related strong CP problem, whose solution in this approach should also follow from some gauge symmetry. Otherwise, it would be highly sensitive to Planck scale physics too, as is, for example, the Peccei-Quinn solution [41. Another open question is whether the gauge couplings and gauge groups may unify at some high scale. Finally, the vacuum in our model is metastable (with a lifetime longer than the age of the universe [42]), and this raises the question why it was chosen by the early universe.

Acknowledgements: We would like to thank M. Luty and S. Willenbrock for discussions. Fermilab is operated by the URA under DOE contract DE-AC02-76CH03000.

\section{Appendix A: The 4-3 model}

The detailed discussion of SUSY breaking in the $S U(4) \times S U(3)$ model can be found in Refs. [15, 16, 6]. Here we just present the model with the $U(1)_{\mu}$ charge assignment, and a brief description of the essential results. The field content and the $U(1)_{\mu}$ charges are shown in Table 6 .

The superpotential of the DSB sector is given by

$$
W_{D S B}=\lambda_{1} \mathcal{L}_{1} \mathcal{Q} \mathcal{R}_{1}+\lambda_{2} \mathcal{L}_{2} \mathcal{Q} \mathcal{R}_{2}+\lambda_{3} \mathcal{L}_{3} \mathcal{Q} \mathcal{R}_{3}+\frac{\alpha}{3 !} \mathcal{R}_{1} \mathcal{R}_{2} \mathcal{R}_{4}
$$

We assume that $\alpha \ll \lambda_{1}, \lambda_{2}, \lambda_{3} \sim 1$, so that the vacuum lies in the weakly coupled regime and hence calculable. The low energy degrees of freedom can be described by the 


\begin{tabular}{|c||c|c||c|}
\hline Fields & $S U(4)$ & $S U(3)$ & $U(1)_{\mu}$ \\
\hline \hline $\mathcal{Q}$ & 4 & 3 & $-\left(z_{b_{1}}+z_{b_{2}}\right) / 12$ \\
\hline $\mathcal{L}_{1}$ & $\overline{4}$ & 1 & $\left(3 z_{b_{1}}-z_{b_{2}}\right) / 4$ \\
\hline $\mathcal{L}_{2}$ & $\overline{4}$ & 1 & $\left(3 z_{b_{2}}-z_{b_{1}}\right) / 4$ \\
\hline $\mathcal{L}_{3}$ & $\overline{4}$ & 1 & $-\left(z_{b_{1}}+z_{b_{2}}\right) / 4$ \\
\hline $\mathcal{R}_{1}$ & 1 & $\overline{3}$ & $\left(-2 z_{b_{1}}+z_{b_{1}}\right) / 3$ \\
\hline $\mathcal{R}_{2}$ & 1 & $\overline{3}$ & $\left(z_{b_{1}}-2 z_{b_{2}}\right) / 3$ \\
\hline $\mathcal{R}_{3}, \mathcal{R}_{4}$ & 1 & $\overline{3}$ & $\left(z_{b_{1}}+z_{b_{2}}\right) / 3$ \\
\hline
\end{tabular}

Table 6: Particle content and charge assignments in the DSB sector.

baryons $b_{i}$, where

$$
b_{i}=\frac{1}{3 !} \epsilon_{i j k l} \mathcal{R}_{j} \mathcal{R}_{k} \mathcal{R}_{l},
$$

with $U(1)_{\mu}$ charges $z_{b_{1}}, z_{b_{2}}, 0,0$, respectively.

The $b_{3}$ and $b_{4}$ fields get vevs of the order $\left(\alpha^{-\frac{4}{9}} \Lambda_{D}\right)^{3}$, where $\Lambda_{D}$ represent the $S U(4)$ scale.The energy density at the minimum and the masses of the scalar components of $b_{1}, b_{2}$ are

$$
\begin{aligned}
E_{\mathrm{vac}}^{4} & \sim \alpha^{\frac{2}{9}} \Lambda_{D}^{4}, \\
m_{b_{1,2}}^{2} \equiv m_{b}^{2} & \sim \alpha^{\frac{10}{9}} \Lambda_{D}^{2} .
\end{aligned}
$$

At one loop, the $b_{1}$ and $b_{2}$ fields will generate a Fayet-Illiopoulos $D$ term for the $U(1)_{\mu}$ gauge group,

$$
-\xi^{2}=-\sum_{j=1,2} \frac{g_{\mu}^{2}}{16 \pi^{2}} z_{b_{j}} m_{b_{j}}^{2} \ln \frac{M_{V}^{2}}{p^{2}},
$$

where $M_{V}$ represents the mass scale of the heavy fields in the DSB sector, and the lower cutoff scale $p^{2}$ is the larger one between the $U(1)_{\mu}$ breaking scale, $M_{\mu}^{2}$, and $m_{b}^{2}$. They also generate a negative contribution to the mass squared of each scalar field charged under $U(1)_{\mu}$ at two-loop, proportional to the field's charge squared,

$$
\frac{m_{i}^{2}}{z_{i}^{2}} \equiv-\tilde{m}^{2}=-\sum_{j=1,2} 4\left(\frac{g_{\mu}^{2}}{16 \pi^{2}}\right)^{2}\left(z_{b_{j}}\right)^{2} m_{b_{j}}^{2} \ln \frac{M_{V}^{2}}{p^{2}} .
$$

Note that the formulae (A.5), (A.6) only apply when $p^{2}<M_{V}^{2}$. If the $U(1)_{\mu}$ breaking scale $\left(p^{2}=M_{\mu}^{2}\right)$ is higher than $M_{V}^{2}$, the results will be suppressed by a factor $M_{V}^{2} / M_{\mu}^{2}$. 


\section{References}

[1] S. Giddings and A. Strominger, Nucl. Phys. B307 (1988) 854;

S. Coleman, Nucl. Phys. B310 (1988) 643;

G. Gilbert, Nucl. Phys. B328 (1989) 159.

[2] R. Kallosh, A. Linde, D. Linde and L. Susskind, Phys. Rev. D52 (1995) 912; hepth/9502069.

[3] T. Banks and M. Dine, Nucl. Phys. B479 (1996) 173, hep-th/9605136;

A. E. Faraggi, Phys. Lett. B398 (1997) 88, hep-ph/9611219.

[4] S. Weinberg, Phys. Rev. D26, 287 (1982).

[5] J. E. Kim and H. P. Nilles, Phys. Lett. B138, 150 (1984);

D. Suematsu and Y. Yamagishi, Int. J. Mod. Phys. A10, 4521 (1995);

M. Cvetič and P. Langacker, Phys. Rev. D54, 3570 (1996), and Mod. Phys. Lett. 11A, 1247 (1996);

V. Jain and R. Shrock, Phys. Lett. B352, 83 (1995) and ITP-SB-95-22, hepph/9507238;

Y. Nir, Phys. Lett. B354, 107 (1995);

M. Cvetič, D. A. Demir, J. R. Espinosa, L. Everett, P. Langacker, Phys. Rev. D56, 2861 (1997), hep-ph/9703317.

[6] H.-C. Cheng, B. A. Dobrescu and K. T. Matchev, FERMILAB-PUB-98/205-T, hepph/9807246, to be published in Phys. Lett. B.

[7] R. Mohapatra and S. Nandi, Phys. Rev. lett. 79, 181 (1997), hep-ph/9702291;

Z. Chacko, B. Dutta, R. Mohapatra and S. Nandi, Phys. Rev. D56, 5466 (1997), hep-ph/9704307.

[8] B. Dobrescu, Phys. Lett. B403, 285 (1997), hep-ph/9703390.

[9] C. D. Froggatt and H. B. Nielsen, Nucl. Phys. B147, 277 (1979).

[10] Super-Kamiokande Collaboration (Y. Fukuda et al.), Phys. Rev. Lett. 81, 1562 (1998), hep-ex/9807003 
[11] M. Dine and A. E. Nelson, Phys. Rev. D48, 1277 (1993), hep-ph/9303230;

M. Dine, A. Nelson, Y. Nir, and Y. Shirman, Phys. Rev. D53, 2658 (1996), hep$\mathrm{ph} / 9507378$.

[12] M. Dine, A. Nelson, and Y. Shirman, Phys. Rev. D51, 1362 (1995), hep-ph/9408384.

[13] G. 't Hooft, Recent Developments in Gauge Theories (Plenum Press, 1980) 135; reprinted in Unity of Forces in the Universe Vol. II, A. Zee ed. (World Scientific 1982) 1004.

[14] I. Affleck, M. Dine, and N. Seiberg, Nucl. Phys. B256, 557 (1985).

[15] E. Poppitz, Y. Shadmi, and S. P. Trivedi, Nucl. Phys. B480, 125 (1996), hepth/9605113.

[16] N. Arkani-Hamed, J. March-Russell, and H. Murayama, Nucl. Phys. B509, 3 (1998), hep-ph/9701286.

[17] S. Dimopoulos, G. F. Guidice, and A. Pomarol, Phys. Lett. B389, 37 (1996), hep$\mathrm{ph} / 9607225$.

[18] K. Choi, E. J. Chun and J. S. Lee, Phys. Rev. D55, 3924 (1997), hep-ph/9611285.

[19] S. Martin, Phys. Rev. D55, 3177 (1997), hep-ph/9608224.

[20] P. Fayet, Nucl. Phys. B90, 104 (1975);

R. Kaul and P. Majumdar, Nucl. Phys. B199, 36 (1982);

H. Haber and G. Kane, Phys. Rep. 117, 75 (1985).

[21] L. Randall and R. Sundrum, preprint MIT-CTP-2788; hep-ph/9810155.

[22] G. Giudice, M. Luty, H. Murayama and R. Rattazzi, preprint CERN-TH/98-337; hep-ph/9810442.

[23] D. Pierce and A. Papadopoulos, Nucl. Phys. B430, 278 (1994), hep-ph/9403240, and Phys. Rev. D50, 565 (1994), hep-ph/9312248.

[24] J. Bagger, K. Matchev, D. Pierce and R.-J. Zhang, Nucl. Phys. B491, 3 (1997), hep-ph/9606211.

[25] C.-H. Chen, M. Drees and J. Gunion, Phys. Rev. D55, 330 (1997), hep-ph/9607421; Phys. Rev. Lett. 76, 2002 (1996), hep-ph/9512230. 
[26] S. Dimopoulos, M. Dine, S. Raby, and S. Thomas, Phys. Rev. Lett. 76, 3494 (1996), hep-ph/9601367;

D. R. Stump, M. Wiest, and C. P. Yuan, Phys. Rev. D54, 1936 (1996), hep$\mathrm{ph} / 9601362$;

S. Ambrosanio, G. L. Kane, G. D. Kribs, S. P. Martin, and S. Mrenna, Phys. Rev. Lett. 76, 3498 (1996), hep-ph/9602239; Phys. Rev. D54, 5395 (1996), hep-ph/9605398;

S. Dimopoulos, S. Thomas, and J. D. Wells, Phys. Rev. D54, 3283 (1996), hepph/9604452; Nucl. Phys. B488, 39 (1997), hep-ph/9609434;

K. S. Babu, C. Kolda, and F. Wilczek, Phys. Rev. Lett. 77, 3070 (1996), hepph/9605408;

J. Bagger, K. Matchev, D. Pierce and R.-J. Zhang, Phys. Rev. D55, 3188 (1997), hep-ph/9609444;

S. Ambrosanio, G. D. Kribs, and S. P. Martin, Phys. Rev. D56, 1761 (1997), hepph/9703211;

H. Baer, M. Brhlik, C.-H. Chen and X. Tata, Phys. Rev. D55, 4463 (1998), hepph/9706509;

C.-H. Chen and J. Gunion, Phys. Rev. D58, 075005 (1998), hep-ph/9802252.

[27] J. Feng and T. Moroi, Phys. Rev. D58 035001 (1998), hep-ph/9712499.

[28] D. A. Dicus, B. Dutta, and S. Nandi, Phys. Rev. Lett. 78, 3055 (1997), hepph/9701341;

D. J. Muller and S. Nandi, preprint OSU-HEP-98-8, hep-ph/9811248.

[29] K. Maeshima, preprint FERMILAB-CONF-96/412-E.

[30] C. H. Chen, J. Gunion, Phys. Lett. B420, 77 (1998), hep-ph/9707302, and the last reference in [26].

[31] F. Abe et al., Phys. Rev. D58, 051102 (1998), hep-ex/9805017.

[32] M. Dittmar and H. Dreiner, Phys. Rev. D55, 167 (1997), hep-ph/9608317.

[33] T. Han and R.-J. Zhang, preprint MADPH-98-1067, hep-ph/9807424.

[34] A. Turcot, talk presented at the Higgs working group meeting, September 25, 1998, Fermilab; summary available at http://fnth37.fnal.gov/higgs/turcot.html. 
[35] B. Abbot et al., Phys. Rev. Lett. 80, 442 (1998), hep-ex/9708005;

F. Abe et al., Phys. Rev. Lett. 81, 1791 (1998), hep-ex/9801019;

F. Abe et al., preprint FERMILAB-PUB-98/206-E, Subm. to Phys. Rev. D, hepex/9806034.

[36] B. Abbott et al., Phys. Rev. Lett. 80, 1591 (1998), hep-ex/9705015;

F. Abe et al., Phys. Rev. Lett. 80, 5275 (1998), hep-ex/9803015.

[37] F. Abe et al., Phys. Rev. Lett. 76, 2006 (1996);

S. Abachi et al., preprint FERMILAB-CONF-96/254-E.

[38] K. Matchev, talk presented at SUSY-98, Oxford, England.

[39] H. Haber and D. Wyler, Nucl. Phys. B323, 267 (1989);

F. Franke and H. Fraas, Z. Phys. C72, 309 (1996);

U. Ellwanger and C. Hugonie, Eur. Phys. J. C5, 723 (1998).

[40] S. Thomas, Talk given at 5th International Conference on Supersymmetries in Physics (SUSY 97), Philadelphia, PA, 27-31 May 1997; K. I. Izawa, Y. Nomura, K. Tobe, T. Yanagida, Phys. Rev. D56, 2886 (1997), hep-ph/9705228; Y. Nomura and K. Tobe, UT-784, hep-ph/9708377; A. Nelson and M. J. Strassler, UW-PT/9808, hep-ph/9806346.

[41] H. Georgi, L. J. Hall and M. B. Wise, Nucl. Phys. B 192 (1981) 409;

M. Dine and N. Seiberg, Nucl. Phys. B 273 (1986) 109;

M. Kamionkowski and J. March-Russell, Phys. Lett. B 282 (1992) 137, hepph/9202003;

S. Ghigna, S. M. Lusignoli and M. Roncadelli, Phys. Lett. B 283 (1992) 278;

R. Holman, S. D. H. Hsu, T. W. Kephart, E. W. Kolb, R. Watkins and L. M. Widrow, Phys. Lett. B 282 (1992) 132, hep-ph/9203206;

S. Barr and D. Seckel, Phys. Rev. D 46 (1992) 539;

B. A. Dobrescu, Phys. Rev. D55 (1997) 5826, hep-ph/9609221.

[42] I. Dasgupta, B. A. Dobrescu, and L. Randall, Nucl. Phys. B483, 95 (1997), hep$\mathrm{ph} / 9607487$. 